\title{
Two isoleucyl tRNAs that decode 'synonymous' codons divergently regulate breast cancer progression
}

Lisa B. Earnest-Noble ${ }^{1}$, Dennis Hsuㄹ, Hosseinali Asgharian ${ }^{2-3}$, Mandayam Nandan ${ }^{1}$, Maria C. Passarelli ${ }^{1}$, Hani Goodarzi ${ }^{2-4 *}$, Sohail F. Tavazoie ${ }^{1, *}$

${ }^{1}$ Laboratory of Systems Cancer Biology, The Rockefeller University, 1230 York Avenue, New York, NY 10065, USA.

2 Department of Biochemistry and Biophysics, ${ }^{3}$ Urology, and ${ }^{4}$ Helen Diller Family Comprehensive Cancer Center, University of California, San Francisco, San Francisco, CA 94158, USA

* To whom correspondence should be addressed. E-mail: stavazoie@rockefeller.edu and hani.goodarzi@ucsf.edu 
1 The human genome contains 61 codons that encode for the $\mathbf{2 0}$ amino acids. 2 The synonymous codons of a given amino acid are decoded by a set of 3 transfer RNAs (tRNAs) called isoacceptors. We report the surprising 4 observation that two isoacceptor tRNAs that decode synonymous codons 5 are modulated in opposing directions during breast cancer progression. 6 Specifically, tRNA ${ }^{l l e}$ UAU is upregulated, whereas tRNA $A^{l l e}{ }_{\text {GAU }}$ is repressed as 7 breast cancer cells attained enhanced metastatic capacity. Functional 8 studies revealed that tRNA ${ }^{\text {Ile }}$ UAU promoted and tRNA ${ }^{\text {lle }}{ }_{\text {GAU }}$ suppressed 9 metastatic colonization. The expression of these tRNAs mediated opposing 10 effects on codon-dependent translation of growth promoting genes. Consistent with this, multiple mitotic gene sets in the human genome are 12 significantly enriched in the codon cognate to the growth-promoting 13 tRNA ${ }^{I l e}$ UAU and significantly depleted of the codon cognate to the growthsuppressive tRNA ${ }^{\| l e}$ GAU. Our findings uncover a specific isoacceptor tRNA pair that act in opposition-divergently regulating genes that contribute to growth and a disease phenotype. The degeneracy of the genetic code can thus be biologically exploited by human cancer cells via tRNA isoacceptor shifts that facilitate the transition towards a growth-promoting state. 
Main

Because of the degeneracy of the genetic code, multiple transfer RNAs (tRNAs) bearing distinct anticodons can accept the same amino acid for translational incorporation into the growing polypeptide chain during translation ${ }^{1,2}$. Such tRNA isoacceptors recognize what are called 'synonymous codons'. Transfer RNAs have long been considered static adaptor molecules that play critical roles in converting the genetic code to an amino acid code. This notion has been revisited in recent years with observations of altered expression of tRNAs in the context of disease ${ }^{3-5}$, as well as demonstrated roles for certain over-expressed tRNAs (by genomic amplifications) as promoters of tumourigenic phenotypes ${ }^{3,4,6}$. Analogous to these observations, aminoacyl tRNA synthetases (aaRS), responsible for charging tRNAs with cognate amino acids, have been shown to play non-canonical roles $^{7}$ and recent work has demonstrated significant cancer progression roles for specific charging enzymes ${ }^{8,9}$. These studies have raised a number of questions, including whether transcriptional deregulation in the absence of tRNA genomic copy number alterations can modulate tRNA levels and cancer progression as well as whether there exist metastasis suppressor tRNAs in human cancer.

\section{Isoleucyl tRNA isoacceptors are divergently modulated in breast cancer}

To identify tRNAs that may become transcriptionally modulated during cancer progression, we performed chromatin immunoprecipitation sequencing (ChIP-seq) in poorly and highly metastatic human breast cancer cells ${ }^{10}$ using an antibody targeting the DNA binding subunit of Polymerase III, POLR3A. Enrichment of tRNA loci was confirmed by successful co-immunoprecipitation of Pol III genomic target loci through quantitative real-time PCR (qPCR) (Supplementary Fig. 1a), as well as significant enrichment of ChIP-seq reads for tRNA Box A and Box B gene regulatory sequences (Supplementary Fig. 1b). We observed that an isoleucyltRNA (TAT) isoacceptor locus that encodes tRNA ${ }^{\text {lle }}$ UAU was significantly more bound by Pol III in highly metastatic MDA- LM2 cells relative to the parental poorlymetastatic MDA-MB-231 cells from which it was derived (Fig. 1a). To confirm these findings and to establish that mature tRNA ${ }^{1 e_{U A U}}$ levels are upregulated in metastatic cells, we performed targeted tRNA profiling by tRNA Capture-seq ${ }^{4}$. Targeted tRNA quantification in the MDA-MB-231 poorly/highly metastatic pair as well as an independent poorly/highly metastatic isogenic human breast cancer line pair (HCC1806-Par and HCC1806-LM2C, validated in Supplementary Fig. 1c) confirmed that mature tRNA ${ }^{11 \mathrm{Ue}}$ UAU is upregulated in highly metastatic breast cancer cells relative to isogenic poorly metastatic cells (Fig. 1b). Northern blot analysis confirmed the observations of tRNA ${ }^{\text {Ile }}$ UAU over-expression in highly metastatic breast cancer cells (Supplementary Fig. 1d). Genomic copy number analysis by qPCR did not reveal increased genomic copy number of isoleucyl-tRNA (TAT) loci in highly metastatic cells, consistent with transcriptional enhancement (Supplementary Fig. 1e). In parallel to these observations, we made the surprising observation that one of the other isoacceptors of isoleucine, tRNA ${ }^{1 \mathrm{le}}{ }_{\mathrm{GAU}}$, became significantly repressed in the highly metastatic sublines relative to isogenic poorly metastatic parental cells (Fig 1c). The high sequence similarity between tRNA ${ }^{\text {lle }}{ }_{\text {GAU }}$ 
92 and another isoleucine isoacceptor tRNA ${ }^{\text {lle }} \mathrm{AAU}$ precluded specific northern blot 93 quantification for tRNA ${ }^{\text {lle }}$ GAU as an independent tRNA quantification method. We 94 thus employed pre-tRNA quantification as an orthogonal approach for assessing the levels of all three isoleucyl tRNAs. Pre-tRNA qRT-PCR also revealed upregulation of tRNA ${ }^{\text {lle }}$ UAU expression by the multiple genomic loci that encode it and conversely, repression of tRNA ${ }^{l l e}{ }_{G A U}$ loci genes in both pairs of highly metastatic breast cancer cells relative to their isogenic poorly metastatic parental cell populations (Fig 1d-e). We did not observe such global modulations of the third isoleucyl isoacceptor pre-tRNA ${ }^{\text {lle }}$ AAU across the loci surveyed (Supplementary Fig.

100 1f). In support of these findings, FISH staining of human tissue microarrays of

102 breast cancer patients with locked nucleic acids (LNAs) targeting tRNAlle UAU and

103 tRNA $^{\text {lle }}$ GAU revealed a significantly increased ratio of tRNA ${ }^{l l e}$ UAU $_{\text {tRNA }}{ }^{l l e}$ GAU

104 expression in stage III breast tumours, which exhibit higher rates of metastatic

105 relapse, relative to stage I or stage II tumours, which exhibit lower rates of

106

107 metastasis (Fig. 1f). These findings reveal that metastatic progression in breast cancer selects for upregulation of one isoleucyl tRNA isoacceptor and repression of another. This shift in tRNA isoleucyl isoacceptor levels suggests potentially differential roles for these tRNAs in breast cancer progression.

\section{TRNA ${ }^{l l e}$ UAU promotes and tRNA ${ }^{l l e}$ GAU suppresses breast cancer metastasis}

To determine if the observed reciprocal tRNA isoleucyl isoacceptor modulations play causal roles in cancer progression, we performed loss-of-function and gainof-function studies for these tRNA isoacceptors. We first sought to overexpress tRNA $^{l l e}$ UAU in poorly metastatic cells to assess whether its upregulation was sufficient to confer increased metastatic capacity (Supplementary Fig. 2a-b). Stable over-expression of tRNA ${ }^{\text {lle }}$ UAU to pathophysiologically relevant levels ( $50 \%$ increase) in poorly metastatic MDA-MB-231 or HCC1806 human cell lines significantly increased lung metastatic colonization in tail-vein colonization assays as assessed by bioluminescence imaging and histological analyses (Fig. 2a-b). For loss-of-function studies, we employed CRISPR-Cas9 using two independent guides specific to tRNA ${ }^{l l e}$ UAU genomic loci (Supplementary Fig. 2c). CRISPR-Cas9 mediated depletion of tRNA ${ }^{l l e}$ UAU in highly metastatic MDA-LM2 breast cancer cells to levels similar to poorly metastatic cells was sufficient to significantly impair breast cancer metastatic colonization (Fig. 2c). These findings reveal tRNA ${ }^{\text {lle }}$ UAU to be a promoter of metastatic progression in these human breast cancer cells.

We next determined if tRNA ${ }^{l l e}{ }_{G A U}$, which became repressed in metastatic cells, plays a causal role in breast cancer progression. TRNA ${ }^{\text {lle }}$ GAU was stably overexpressed in highly metastatic MDA-LM2 cells to levels similar to those observed in poorly metastatic MDA-231 parental cells ( 1.8-fold over-expression) (Supplementary Fig. 2d). Increasing tRNA ${ }^{\text {lle }} \mathrm{GAU}$ expression in highly metastatic MDA-LM2 cells substantially reduced metastatic lung colonization capacity (Fig. $1342 \mathrm{~d}$ ). Given the high sequence similarity between tRNA ${ }^{\text {lle }}$ GAU and ${ }^{2}$ RNA ${ }^{\text {lle }}$ AAU, we 135 employed two orthogonal approaches for tRNA ${ }^{\text {lle }}$ GAU loss-of-function-CRISPRi 136 and shRNA mediated interference. Firstly, MDA-231 cells were stably transduced 137 with mutant Cas9-KRAB and a specific guide complementary to common 
138 sequences in tRNA ${ }^{\text {lle }}$ GAU genomic loci. Reduced tRNA ${ }^{l l e}$ GAU was confirmed by

139 targeted tRNA capture qPCR (Supplementary Fig. 2e). ShRNA-mediated 140 interference was also employed using a hairpin specific to tRNA ${ }^{\text {ile }}$ GAU 141 (Supplementary Fig. 2f). Depletion of tRNA ${ }^{l l e}$ GAU using both approaches enhanced 142 lung metastatic colonization by poorly metastatic MDA-231 cells (Fig. 2e and 2f). 143 These findings implicate tRNA ${ }^{\text {lle }}$ GAU as a metastasis suppressor tRNA and uncover 144 two surprising findings: the first being a gain-of-function organismal disease 145 phenotype upon depletion of a tRNA (tRNA ${ }^{l l e} G A U$ ); the second being the 146 observation of a dichotomy between two tRNA isoacceptors in regulating a 147 common phenotype.

148 TRNAlle isoacceptors divergently regulate growth and growth gene

\section{expression}

We next sought to identify the cancer progression cellular phenotype(s) regulated by tRNA ${ }^{\mathrm{Ne}}$ UAU and tRNA ${ }^{\mathrm{ll}}{ }_{\text {GAU }}$ by searching for gene sets that exhibit enrichments or depletions of codons cognate to these tRNAs. We performed pathway enrichment analyses using the iPAGE framework ${ }^{11}$-assessing significant genome-wide abundances for the codons cognate to tRNA ${ }^{1 \mathrm{ll}}$ UAU and TRNA ${ }^{1 \mathrm{le}}$ GAU in an unbiased manner. All coding transcripts in the human genome were ranked and binned by AUA or AUC relative synonymous codon usage (RSCU). Pathways that were significantly enriched $\left(p<10^{-3}\right)$ across discretized bins were identified based on their mutual information content (Fig. 3a-b). Interestingly, transcripts most signficantly enriched in AUA codons (cognate to tRNA ${ }^{\text {IIe }}$ UAU) were enriched in mitosis related gene sets such as metaphase, anaphase, and chromatid separation, and homologous DNA pairing and strand exchange (Fig. 3a). Conversely, AUC codons (cognate to tRNA ${ }^{l l}{ }_{G A U}$ ) were most significantly depleted from these mitosis related gene sets (Fig. 3b). As an orthogonal and functional approach for identifying the downstream consequences of modulation of these tRNAs, we conducted ribosomal profiling of breast cancer cells in the context of tRNA ${ }^{l l}$ UAU overexpression and tRNAlle GAU depletion (by CRISPRi), mirroring the divergent tRNA ${ }^{\text {lle }}$ modulations observed in highly metastatic cells relative to poorly metastatic cells. Ribosomal protected fragments were sequenced, and conformed to the expected size and periodicity reported by other groups ${ }^{12}$ (Supplementary Fig. 3a-b). Ribosomal occupancy of transcripts was then quantified as a measure of translational efficiency (Supplementary Fig 3c). Genes enriched in GO terms such as cell cycle and mitosis exhibited enhanced translational efficiency (Fig. 3c). At the proteomic level, GO functional analysis of proteins in tRNA ${ }^{11}{ }_{U A U} /$ tRNA ${ }^{110}{ }_{G A U}$ modulated cells by label free mass spectrometric quantification also revealed enrichment of gene sets including cell cycle, mitosis, as well as regulation of stress response relative to control cells (Fig. 3d). Consistent with the growth related gene sets identified using the described approaches, immunofluorescent staining of metastatic nodules for the proliferation marker Ki67 revealed that MDA MB 231 breast cancer cells concomitantly over-expressing tRNA ${ }^{\text {lle }}$ UAU and depleted of tRNA ${ }^{1 l}{ }_{\text {GAU }}$ exhibited greater proliferation than control cells (Fig. 3e, Supplementary Fig. 3d). To determine if these in vivo observations could be recapitulated in vitro, growth assays were performed under normal tissue culture conditions and under 
184 conditions of hypoxic and oxidative stress, since such stresses occur in the 185 metastatic microenvironment and can restrict growth ${ }^{13-18}$. Concomitant tRNA ${ }^{\text {lle }}$ UAU 186 upregulation/tRNA ${ }^{\text {lle }}$ GAU depletion enhanced the in vitro growth of MDA MB 231 187 breast cancer cells relative to control cells in the context of hypoxia (Fig. $3 \mathrm{f}$ ) and 188 oxidative stress (Fig. $3 g$ ). Importantly, growth effects were more pronounced under 189 these stress conditions that are known to occur in the tumour microenvironment 190 than under normoxic basal in vitro conditions (Supplementary Fig. 3e). These 191 findings reveal that divergent modulation of these isoleucyl tRNA isoacceptors 192 promotes growth in these breast cancer cells in vivo and in vitro.

\section{A growth gene network regulated by TRNAlle isoacceptors}

We next sought to identify examples of downstream effector genes that could mediate cell growth effects downstream of tRNA ${ }^{\mathrm{lle}}{ }_{\text {UAU }} / \mathrm{RNA} \mathrm{Al}_{\mathrm{GAU}}^{\mathrm{lle}}$ modulation. We hypothesized that there exist growth-promoting genes enriched in AUA codons cognate to tRNA $A^{\| l}$ UAU. We identified the set of genes that exhibited enhanced translational efficiency as well as enhanced mass-spectrometric protein abundances upon concurrent tRNA ${ }^{\mathrm{Ile}}$ UAU/tRNA ${ }^{\mathrm{Ile}}{ }_{\text {GAU }}$ modulation, and exhibited a high relative synonymous codon usage score for $t R N A^{\| l e}$ UAU. The ten genes that fulfilled these criteria were further restricted to those that exhibited enhanced translational efficiencies and protein abundances in highly metastatic cells, which endogenously modulate these tRNAs relative to the isogenic parental poorly metastatic population (Fig. $3 \mathrm{~h})^{4}$. This yielded six genes as candidate downstream growth-promoting effectors (Supplementary Fig. 3f). Functional testing revealed that RNAi-mediated depletion of three of these genes (SMNDC1, LSM6, and PYCARD) reduced proliferation (3i-k, Supplementary Fig. $3 g, h$ ). We next focused on one gene, SMNDC1, for mutagenesis studies. To determine if isoleucyl tRNA modulations can directly enhance translation of a growth-promoting gene in a codon-dependent manner, we employed a reporter-based approach in which AUA codons in SMNDC1 were mutated to synonymous AUC codons. While the wildtype SMNDC1 protein became upregulated upon dual tRNA modulation, synonymous codon mutant SMNDC1 protein levels remained unchanged (Fig. 3l, m)consistent with codon-dependent tRNA ${ }^{\text {lle }}$ UAU-driven enhancement of translation of this growth-promoting gene. These findings reveal that divergent isoleucyl tRNA modulation enhances translation of a set of growth-promoting genes with high tRNA ${ }^{l l e}$ UAU relative synonymous codon usage scores.

\section{Divergent tRNA isoacceptor modulation impacts ribosomal function}

The opposing directionality of the metastasis phenotype observed upon modulating these isoacceptor tRNAs suggests that they may elicit distinct downstream codon-dependent translational effects at a global level. To test this, we performed polysome profiling studies (Supplementary Fig. 4a). This revealed that relative to control cells, concurrent tRNA ${ }^{1 \mathrm{le}}$ UAU over-expression and tRNA ${ }^{1 \mathrm{le}}$ GAU depletion elicited a significant increase in polysome occupancy of transcripts enriched in the AUA codon, which is cognate to the over-expressed tRNA ${ }^{l l e}$ UAU (zscore 23.6; robustness 10/10; Fig. 4a) and a reduction in actively translating 
230 transcripts enriched in the AUC codon, which is cognate to the depleted tRNA ${ }^{l l e}$ GAU 231 (z-score 44.8; robustness 10/10; Fig. 4b). Consistent with this, analysis of the 232 aforementioned ribosomal profiling data revealed that upon dual tRNA $^{\text {lle }}$ UAU/tRNA ${ }^{\text {ile }}$ GAU modulation, there was also a significant enrichment of ribosomal occupancy of AUA-containing transcripts and reduced occupancy of AUC-containing transcripts (Supplementary Fig. 4b,c). Our findings as a whole suggest a model whereby tRNA ${ }^{l l e} \mathrm{UAU}_{\mathrm{T}} / \mathrm{RNA} \mathrm{Al}_{\mathrm{GAU}}$ modulation enhances the efficiency of AUA codon decoding by the ribosome. This would suggest that we should observe reduced ribosomal dwell time over AUA codons upon dual tRNA modulation. Moreover, we would expect to see increased binding of tRNA ${ }^{l l e}$ UAU relative to $\mathrm{tRNA}^{\mathrm{lle}} \mathrm{GAU}$ to the ribosome upon tRNA ${ }^{\mathrm{lle}} \mathrm{UAU}_{\mathrm{UU}} / \mathrm{tRNA}{ }^{l l e} \mathrm{GAU}$ modulation. In order to capture the dwell time of ribosome at every codon, we measured the extent to which its occupancy in the ribosome profiling data deviates from its predicted level based on loess regression ${ }^{19}$. We observed that tRNA $^{\text {lle }}{ }_{\text {UAU/tRNA }}{ }^{\text {lle }}$ GAU modulation significantly reduced ribosome dwell time over AUA codons, consistent with productive translation, while over-expression of tRNA ${ }^{l l e}$ UAU or depletion of tRNA ${ }^{\text {lle }}$ GAU individually were insufficient to elicit significant shifts in dwell time (Fig. 4c). To determine if tRNA modulations impact ribosomeassociated tRNA ${ }^{\text {lle }}$ UAU and tRNA ${ }^{l l e}$ GAU abundances, we quantified the abundance of these tRNAs from polysomal ribosomes as well as total cellular input. We observed that tRNA ${ }^{l l e}$ GAU depletion reduced the ribosomal association of this tRNA, while tRNA ${ }^{l l e}$ UAU over-expression enhanced its ribosome association (Fig. 4f). Importantly, dual tRNA ${ }^{\text {lle }}$ UAU/tRNA ${ }^{\text {ile }}$ GAU modulation caused the greatest increase in relative tRNA ${ }^{\text {lle }}$ UAU to tRNA ${ }^{\text {lle }}$ GAU ribosomal association (Fig. 4g). The substantially increased ribosomal association of tRNA ${ }^{\text {lle }}$ UAU upon dual tRNA ${ }^{\text {lle }}$ UAU/tRNA ${ }^{\text {lle }}$ GAU modulation relative to $\operatorname{tRNA}_{\mathrm{GAU}}^{\mathrm{lle}}$ depletion supports the translational consequences observed upon polysome profiling (Fig. 4d, e). These concordant observations of global shifts in isoleucine codon enrichments and depletions in polysome profiling and ribosomal profiling studies as well as dwell time and biochemical analyses support direct codon-dependent effects on translation upon divergent modulation of these tRNAs. Our findings as a whole support a model whereby isoleucyl isoacceptor tRNA abundance shifts impact codon-dependent translation of growth regulating genes at the ribosome, thereby promoting cancer progression (Fig. 4h).

\section{Discussion}

Our observations reveal opposing roles for two isoleucyl tRNAs in regulation of breast cancer metastatic colonization and cancer cell growth. Our findings as a whole support a model whereby shifts in tRNA lle isoacceptor abundance impact codon-dependent translation of growth regulating genes at the ribosome, thereby promoting cancer progression (Fig. 4h). The molecular and functional studies implicating growth as a phenotype divergently impacted by modulation of these tRNAs is supported by genome sequence analyses that reveal significant enrichment or depletion of the codons cognate to these antagonistic tRNAs in

275 mitotic gene sets. Future studies are warranted to better elucidate the molecular 
276 basis of such interferences and to search for additional examples of such antagonistic isoacceptor tRNA pairs in health and disease.

\section{Methods}

\section{Cell Culture}

283 MDA-MB-231 and its highly metastatic derivative ${ }^{10}$ LM2 cells were cultured with DMEM media supplemented with $10 \%$ FBS, sodium pyruvate, and L-glutamine. HCC1806 Parental and derivate cell lines were cultured in 1x RPMI supplemented with $10 \%$ FBS, sodium pyruvate, $1 \mathrm{mM}$ HEPES as specified by ATCC. All cell lines were regularly tested for mycoplasma infection and were negative. Each cell line was verified using STR testing, performed by the Integrated Genomics Operation at MSKCC. Cells were retrovirally transduced with a luciferase reporter for bioluminescence detection as previously described ${ }^{4,15,20}$. Oxidative stress analyses were conducted by addition of $200 \mathrm{uM}$ hydrogen peroxide to cells.

\section{In Vivo Selection}

Several female Nod SCID Gamma (NSG) (Jackson \# 005557) mice were injected at 6 weeks of age intravenously via tail vein with 150,000 parental HCC1806 cells and monitored by bioluminescence IVIS imaging (IVIS Lumina II) until photon flux of lungs reached $10^{\wedge} 7$ or $10^{\wedge} 8$ (4-7 weeks). Subsequently, animals were euthanized according to IACUC protocol and guidelines, and the lungs were extracted under sterile conditions. The lungs were then placed on a sterile $6 \mathrm{~cm}$ tissue culture dish and minced with razor blades. Lung tissue extracts were resuspended in $20 \mathrm{~mL}$ RPMI Media supplemented with FBS, sodium pyruvate and HEPES with $15 \mathrm{mg} / \mathrm{mL}$ Collagenase IV (Worthington). Cells were incubated at $37^{\circ} \mathrm{C}$ for 30 minutes on a shaker to allow for digestion. Tissue extracts were then spun down at 1000 RPM for 5 minutes at $4^{\circ} \mathrm{C}$ and resuspended in RPMI media without Collagenase IV. Cells were filtered with a 100 um filter (Corning) and spun down again. Cells were then treated with $5 \mathrm{~mL}$ ACK Lysis Buffer and left at room temperature for 5 minutes. Cells were spun again and resuspended in $1 \mathrm{~mL}$ Optiprep solution 1 (2:1, Optiprep:Media). A gradient was constructed with an additional $4 \mathrm{~mL}$ Solution 1, overlain by $3 \mathrm{~mL}$ Optiprep Solution 2 (2.2:1, Solution1: Media). $1 \mathrm{~mL}$ Media was overlain and the gradient was spun down for 20 minutes at 1000 RPM $4^{\circ} \mathrm{C}$. Viable cells were collected from the top of the gradient and washed twice with RPMI media. Cells were then plated in $75 \mathrm{~cm}$ filtered flasks with PenStrep and Fungizone added to the RPMI Media. The cells were cultured for approximately a week to reduce stromal cell survival and then tested for mycoplasma. To generate an in vivo selected line twice, these lung metastatic 1 (LM1) generation cells were then re-injected at 150,000 tail vein and the process was repeated.

\section{Chromatin Immunoprecipitation}

MDA-MB-231 and LM2 cells in biological replicates were plated in $15 \mathrm{~cm}$ plates ( $\sim 12$ million cells). For cross-linking, $1 \%$ formaldehyde was added to cells at $37^{\circ} \mathrm{C}$ 
322 minutes at room temperature. The plates were put on ice, the media was removed 323 and cells were washed with ice cold PBS twice. 500 uL PBS with 1x HALT protease 324 inhibitors (Thermo) were added and cells were scraped and put in an Eppendorf on ice. Cells were pelleted at 4000 RPM in a refrigerated centrifuge for 4 minutes. The cell pellets were then resuspended in $400 \mathrm{uL}$ Lysis buffer (1\% SDS, $50 \mathrm{mM}$ Tris Hcl pH 8.020 mM EDTA, protease inhibitors (Roche) and incubated on ice for 10 minutes. Lysates were then sonicated to produce DNA fragments between 200 - 1000 bp with settings Amplitude 70, 10 sec on 30 sec rest for three repetitions on Sonicator S-4000 (Branson) with Microtip and Ultrasonic Liquid Processor (Misonix). Lysates were kept on ice during sonication to prevent protein degradation. Lysates were then clarified by centrifugation at max speed at $4{ }^{\circ} \mathrm{C}$ for 10 minutes. Equivalent amounts of lysate were then added to separate eppendorfs, saving some lysate for input samples. Either 5 ug rabbit lgG or 5 ug POLR3A (Cell Signaling \#12825S) were then added, with a final volume of $1 \mathrm{ml}$ with protease inhibitors of dilution buffer (16.7 mM Tris $\mathrm{HCl} \mathrm{pH} 8.0,0.01 \%$ SDS, 1.1\% Triton X-100, $1.2 \mathrm{mM}$ EDTA, $167 \mathrm{mM} \mathrm{NaCl})$. Lysate and antibody mixtures were incubated overnight at $4^{\circ} \mathrm{C}$ with rotation. $50 \mathrm{uL}$ Protein $\mathrm{G}$ Dynabeads were added to each sample after washing and incubated at $4{ }^{\circ} \mathrm{C}$ for 2 hours with rotation. Tubes were then placed on a magnet for 2 minutes, discarding the supernatant. The following washes were performed, twice each for 5 minutes at $4{ }^{\circ} \mathrm{C}$ in the following order: low salt (140 mM NaCl, 50 mM HEPES, $0.1 \%$ SDS, $1 \%$ Triton X100, 0.1\% deoxycholate, $1 \mathrm{mM}$ EDTA) high salt (500 mM NaCl, $50 \mathrm{mM}$ HEPES, $0.1 \%$ SDS, $1 \%$ Triton X-100, 0.1\% deoxycholate, 1 mM EDTA), LiCl (250 mM LiCl, $20 \mathrm{mM}$ Tris $\mathrm{HCl} \mathrm{pH} 8.0,0.5 \%$ NP-40, 0.5\% deoxycholate, $1 \mathrm{mM}$ EDTA) and TE Buffer (10 mM Tris HCl pH 8.0, 1 mM EDTA). 100 uL Elution buffer (50 mM Tris $\mathrm{HCl} \mathrm{pH} \mathrm{8.0,} 1 \mathrm{mM}$ EDTA) was then added to the beads and incubated overnight at $65^{\circ} \mathrm{C}$ on a shaker to enable elution. The eluted sample was transferred to a new tube and repeated for a final volume of $200 \mathrm{uL}$ per sample. $1 \mathrm{uL}$ of $10 \mathrm{mg} / \mathrm{mL}$ RNase A was added to each sample (including input samples) and incubated at $37^{\circ} \mathrm{C}$ for 30 minutes. $2 \mathrm{uL}$ of Proteinase $\mathrm{K}$ was added $(10 \mathrm{mg} / \mathrm{mL})$ and incubated for 2 hours at $56^{\circ} \mathrm{C}$. DNA was then purified using the DNA Clean and Concentrator Kit (Zymo Research). Enrichment of Polymerase III bound loci was confirmed with genomic tRNA qPCR primers and quantified as Percent Input over IgG. The Input and IP DNA samples were then PCR amplified with Illumina barcodes to construct a multiplexed library. The library was quantified using TapeStation and sequenced on the 50 SR HiSeq at the Rockefeller Genomics Resource Center.

\section{tRNA Capture qPCR}

tRNA quantification by RT-qPCR was performed as described previously ${ }^{1}$. RNA purified with Norgen Total Purification Kit was quantified using a Nanodrop. Normalized RNA across samples was added to a hybridization mixture (final concentration $10 \mathrm{mM}$ Tris $\mathrm{HCl} \mathrm{pH} 7.4,50 \mathrm{mM} \mathrm{NaCl}, 1 \mathrm{mM}$ EGTA pH 8.0) with 2 uM Hybridization probes. Hybridization probes specific for the following tRNA were used: Ile TAT Left 5 /5Phos/AAGTACCGCGCGCTACCGATTGCGCCACTGGAGCGATCGTCGGAC TGTAGAA, Ile TAT Right 

CGTGTGCTCTTCCGATCTTGCTCCAGGTGAGGCTCGAACTCACACCTCGGC

ATTAT', Ile

GAT

Left

5 15Phos/CAGCACCACGCTCTACCAACTGAGCTAACCGGCCGATCGTCGGACT GTAGAA, Ile GAT

$\mathrm{R}$

TGTTAT 3'. Each 'left' probe contained a 5' phosphate to enable subsequent TGTTAT 3'. Each 'left' probe contained a 5' phosphate to enable subsequent ligation. RNA and probe mixture was hybridized using a thermocycler and brought to RT. 1x SplintR ligase buffer, SplintR Ligase (NEB) and RNase Inhibitor (Promega) were added and incubated at room temperature for 2 hours. An additional ligation step with T4 Ligase was performed overnight at $16^{\circ} \mathrm{C}$. The RNA was then degraded with RNase $A$ (Thermo Fisher) \& $H$ (NEB) for 30 minutes at 37 ${ }^{\circ} \mathrm{C}$. The ligated probe reaction was then diluted 1:50 and quantified using primers (Forward 5' CGTGTGCTCTTCCGATCT 3' \& Reverse 5' GATCGTCGGACTGTAGAA 3') specific to the probe backbone by RT-qPCR. 5S and $18 \mathrm{~S}$ probes were used as loading controls: 5S Left 5' 5PHOS/CTGCTTAGCTTCCGAGATCAGACGAGATCGGGCGCGATCGTCGGA CTGTAGAA $\quad 3$ ' 5 5 $\quad$ Right CGTGTGCTCTTCCGATCTCCAGGCGGTCTCCCATCCAAGTACTAACCAGGC $\begin{array}{lllll}\text { CCGACC } & \text { 3' } & \text { or } & 18 S & \text { Left }\end{array}$ 5PHOS/CCTAGTAGCGACGGGCGGTGTGTACAAAGGGCGCCGATCGTCGGA CTGTAG 3' $18 S$ Right CGTGTGCTCTTCCGATCTCCGATCCGAGGGCCTCACTAAACCATCCAATC 3'.

\section{Northern Blot}

RNA was purified using Norgen total RNA Purification kits according to manufacturer's instructions. 5 ug purified RNA was run on 10\% TBE-Urea gels at 200V for 1 hour, and transferred to a Hybond-N+ membrane (GE) at 150A for 1 hour. RNA was then crosslinked to the membrane at $240 \mathrm{~mJ} / \mathrm{cm}^{2}$, and blocked with Oligo Hybridization Buffer (Ambion) for 1 hour at $42{ }^{\circ} \mathrm{C}$. Northern probes were labeled with ${ }^{32} \mathrm{P}$ ATP with T4 PNK (NEB), purified with a G25 column (GE Healthcare), and hybridized in Oligo Hybridization Buffer overnight at $42{ }^{\circ} \mathrm{C}$. Membranes were washed with $2 X$ SSC $0.1 \%$ SDS Buffer, then with 1 X SSC $0.1 \%$ SDS Buffer. Films were developed at varying times subject to radioactivity of membrane. Probe oligo sequences for lle TAT Intron: 5' ACUGCUGUAUAAGUACCGCGCGC 3 ' and lle TAT 5' cucggcauuauaaguaccgcgcgc 3' and U6 5' CACGAATTTGCGTGTCATCCTT 3'. Membranes were stripped with $0.1 \%$ SDS in boiling water and allowed to cool to room temperature. Quantification was performed with ImageJ and normalized to U6 levels.

\section{RT qPCR}

RNA was purified using the Norgen total RNA Purification kits according to manufacturer's instructions. 1ug purified RNA was used for cDNA production with Superscript III reverse transcriptase (Thermo Fisher Scientific) using random hexamer as a template. The cDNA was diluted 1:5 and quantified with Sybr Green Master Mix (Thermo). The ddCT levels were quantified through normalization to 
$41418 \mathrm{~S}$ with biological replicates. One primer set was used for tRNA ${ }^{\text {lle }}$ GAT genetic loci 415 chr.X-6 and chr.X-7 as their sequences are indistinguishable. Primer sequences 416 are available in the Supplemental Material Section.

\section{tRNA Fluorescence In Situ Hybridization} Breast tissue microarrays were obtained from Biomax (BC08118 \& BR1005b). Slides underwent deparaffination via 5 minute incubations in xylene 2x, 100\% Ethanol, 2x, 70\% Ethanol, 50\% Ethanol, and subsequently molecular grade water. Antigen retrieval was performed with $1 x$ Citrate Buffer $\mathrm{pH} 6.0$ in a microwave for 20 minutes. Slides were cooled to room temperature, then tissue regions were isolated with a PAP pen. Slides were incubated in $0.13 \mathrm{M} 1$-methylimidizaole 300 $\mathrm{mM} \mathrm{NaCl} \mathrm{pH} 8.0$ solution twice for 10 minutes each. Next, slides were incubated with $0.16 \mathrm{M} \mathrm{N}$-(3-Dimethylaminopropyl)-N-ethylcarbodiimide hydrochloride (EDC) (Sigma Aldrich) in the 1-methylimidazole solution for 1 hour at RT to preserve small $\mathrm{RNAs}^{21}$. Slides were then washed with $0.2 \%$ Glycine in Tris buffered saline (TBS) $\mathrm{pH}$ 7.4, then in TBS twice. Pre-hybridization of slides occurred with 1X ISH (Exiqon) buffer at $53^{\circ} \mathrm{C}$ for 1 hour. $40 \mathrm{nM}$ tRNA double DIG labeled LNA Probe targeting tRNA ${ }^{l l e}$ UAU

(Sequence CA+GGTGAGGCTCGAACTCACAC+C+TCGGCAT+T+A 3' with +N indicating LNA at that nucleotide) and tRNAlle ${ }_{G A U}$ (Sequence 5' AGTCGA+GCCCGCGAC+CTTGG+TGTTA+T+C 3') (Qiagen) in 1X ISH buffer was denatured at $95^{\circ} \mathrm{C}$ for 5 minutes followed by cooling on ice for 1 minute. The LNA probe was added to the slide (and covered with a glass coverslip to prevent evaporation) and hybridized overnight at $52^{\circ} \mathrm{C}$. Slides were then washed with $4 \mathrm{X}$ SSC, 2X SSC, 1X SSC, and 0.5X SSC in 50\% formamide for 20 minutes each, then washed with $100 \mathrm{mM}$ Tris- $\mathrm{HCl}$ pH $7.4150 \mathrm{mM} \mathrm{NaCl}$ (TN buffer) for 5 minutes. Slides were blocked with $1 X$ Blocking Reagent (Roche) in TN buffer for 1 hour at RT. Anti-DIG POD in TN blocking buffer was added 1:100 and incubated for 2 hrs. Slides were washed $3 x$ for 5 minutes in TN buffer with $0.05 \%$ Tween-20 (TNT). FITC-tyramide solution 1:100 in 1x amplification reagent (TSA) was incubated on slides for 10 minutes at RT. Slides were subsequently washed $3 x$ for $5 \mathrm{~min}$ with TNT buffer. Samples were washed with PBS and stained with DAPI for 5 minutes, then mounted with Prolong Gold anti-fade solution (Thermo Fisher). Fluorescent intensity was measured on an Inverted TCS SP8 laser scanning confocal microscope (Leica) at the Bioimaging Resource Center at Rockefeller University and quantified by mean fluorescence intensity relative to DAPI. Quantification was performed blind.

\section{Viral Production \& Stable Cell Line Generation}

Stable generation of cell lines was performed as previously described ${ }^{1,6,7}$. Lentivirus was generated using the ViraSafe lentiviral packaging system (Cell Biolabs) with Lipofectamine 2000 (Invitrogen) in HEK293T cells. Transductions were performed with $8 \mathrm{ug} / \mathrm{mL}$ polybrene. Plasmids to overexpress tRNA ${ }^{\text {lle }}$ or with shRNAs targeting tRNA ${ }^{l l e}$ GAU were cloned into the plko.1 puromycin (Addgene \# 8453) or blasticidin (Addgene \#26655) backbone with Agel/EcoRI restriction 459 sites $^{22,23}$. CRISPRi stable cells lines were generated with lentiviral transduction of 
dCas9-KRAB (Addgene \# 110820) and pSLQ plasmid (Addgene \# 51024) cloned with a tRNA ${ }^{\text {lle }}$ GAU targeting guide (5' TGAGCTAACCGGCCGCCCGA 3'), and then flow sorted for positive BFP+ and mCherry+ cells ${ }^{24,25}$. CRISPR generated cells were transduced with lentiCRISPRv2 (Addgene \# 98290) cloned with specific guides targeting tRNA ${ }^{l l e}$ UAU loci (Guide 1: 5' GCGCTAACCGATTGCGCCAC 3', Guide 2: 5' TGGCGCAATCGGTTAGCGCG 3') or the eGFP targeting sequence as control (5' GGGGCGAGGAGCTGTTCACCG 3'). Cells were then either selected with $2 \mathrm{ug} / \mathrm{mL}$ puromycin or $7.5 \mathrm{ug} / \mathrm{mL}$ Blasticidin (Thermo Fisher Scientific).

\section{Animal Studies}

For metastasis assays, tail veins injections were performed in 5-6 week age matched female NOD SCID Gamma mice (The Jackson Laboratory \#005557). Cells were counted via hemacytometer and resuspended in 1x PBS, and 100uL was injected with a $27 G 1 / 2$ needle $(B D)$ into the lateral tail vein. Non-invasive bioluminescence imaging was performed immediately after injections using an IVIS Lumina II (Caliper Life Science) for Day 0 baseline, followed by weekly imaging. Bioluminesence imaging was obtained through retro-orbital injection of 50uL D-luciferin (Perkin Elmer) followed by 1 minute exposure in IVIS Lumina II. Unless otherwise stated, each experimental group consisted of $n=5$ mice. For bioluminescence imaging, cell lines were transduced with triple reporter and FACS sorted for GFP positive cells 48 hours post transduction ${ }^{4,10}$. All animal work was conducted in accordance with protocols approved by the Institutional Animal Care and Use Committee at The Rockefeller University.

\section{Histology}

Lungs were prepared by perfusion fixation with $4 \%$ paraformaldehyde through the circulation via the right ventricle post euthanasia. Lungs were then fixed in $4 \%$ paraformaldehyde overnight at $4^{\circ} \mathrm{C}$. The samples were then embedded in paraffin and sectioned in $5 \mu \mathrm{m}$ slices that were used for immunostaining. $5 \mu \mathrm{m}$ sections at different depths were stained with hematoxylin and eosin (H\&E).

\section{Ribosomal Profiling}

Ribosomal profiling was performed based on the McGlincy \& Ingolia protocol ${ }^{4}$. Briefly, cells were plated in $15 \mathrm{~cm}$ dishes at $50 \%$ confluency the day before collection. The media was aspirated, and the cells were washed with $5 \mathrm{~mL}$ ice cold PBS, and aspirated. The plate was then submerged in liquid nitrogen to freeze the cells. $400 \mathrm{uL}$ ice cold lysis buffer was added to the plate, and scraped immediately. Each lysate was kept on ice until all plates were collected. Several plates were combined with lysis buffer totaling $1 \mathrm{~mL}$ per biological replicate. Lysates were then triturated ten times with a 26 gauge needle. Lysates were clarified at top speed for 10 mins in a cold bench top centrifuge and the supernatant was recovered and snap frozen in liquid nitrogen, then stored at $-80^{\circ} \mathrm{C}$. Lysates were quantified with Quant-iT Ribogreen assay (Life Technologies) and 60 ug total RNA per sample was incubated with 3 uL RNase I (Epicentre \#N6901K) for 45 minutes at RT with

505 light shaking. 10 uL SUPERase*In RNase Inhibitor (Invitrogen) was added to stop 
506 digestion, and the RNA was transferred to a $13 \mathrm{~mm} \times 51 \mathrm{~mm}$ polycarbonate 507 ultracentrifuge tube. $900 \mathrm{uL}$ Sucrose cushion $(1 \mathrm{M}$ Sucrose with $20 \mathrm{U} / \mathrm{mL}$ 508 SUPERase* In in polysome buffer ${ }^{4}$ ) was underlaid and spun at 100,000 RPM at $4^{\circ} \mathrm{C}$ for 1 hour. With ribosomes pelleted, the supernatant was pipetted out of the tube. $300 \mathrm{uL}$ Trizol was added to the pellet and resuspended. RNA was subsequently purified with the Direct-zol kit (Zymo). RNA was then precipitated overnight and resuspended after ethanol washes in $5 \mathrm{uL} 10 \mathrm{mM}$ Tris $\mathrm{HCl} \mathrm{pH} 8.0$. Ribosome footprints were isolated after running a 15\% TBE-Urea gel and the RNA was excised within the range of $17 \mathrm{nt}-34 \mathrm{nt}$ and then precipitated overnight. RNA fragments were then dephosphorylated with T4 PNK and ligated to a DNA linker with T4 Rnl2(tr) K227Q (NEB \#M0351S) for 3 hours with distinct linker barcodes. Unligated linkers were depleted with yeast 5'-deadenylase (NEB \#M0331S) and RecJ exonuclease (Epicentre \#RJ411250) at $30^{\circ} \mathrm{C}$ for 45 minutes. Ligations were then purified with the Oligo Clean \& Concentrater kit (Zymo) and samples were pooled. Ribo Zero Gold was then used to deplete ribosomal RNAs ( 2 reactions were used, and the $50^{\circ} \mathrm{C}$ step was omitted, Illumina). RNA was then purified using Oligo Clean \& Concentration kit. The pooled ligations were then reverse transcribed using Superscript III at $55^{\circ} \mathrm{C}$ for 30 minutes, with RNA templates hydrolyzed by $2.2 \mathrm{uL} 1 \mathrm{M} \mathrm{NaOH}$. Samples were purified with the Oligo Clean \& Concentrator kit and run on a polyacrylamide gel and the RT product was excised above 76nt. Gel slices were incubated with DNA gel extraction buffer overnight after the gel was broken up with gel breaker tubes (IST Engineering) and precipitated overnight. The RT product was resuspended in $10 \mathrm{mM}$ Tris $\mathrm{HCl} \mathrm{pH} 8.0$ and circularized with CircLigase II at $60^{\circ} \mathrm{C}$ for 1 hour. qPCR quantification of circulization products were performed to quantify number of cycles sufficient for library preparation, with the concentration estimated at 713 pM. 8 cycles were used to amplify the library with Pfusion with the primers indicated, NI-799 and NI-7984. Products were purified and size selected at $>136 \mathrm{bp}$, primarily at $160 \mathrm{bp}$. The library was then precipitated, quality checked with Tapestation and sequenced on the NextSeq High Output 75 Single Read at the Rockefeller University Genomics Resource Center. Concurrently 1 ug total RNA was prepped for RNA sequencing according to the manufacturer's instructions (Illumina). Analysis was performed as described previously ${ }^{1}$. For analysis, reads were first subjected to linker removal and quality trimming (cutadapt v1.14). The reads were then aligned against a reference database of rRNAs (iGenomes: AbundantSequenes) and tRNAs

541 (GtRNAdb, hg38) so as to remove contaminants (using bowtie 2.3.4.1). STAR $\mathrm{v}$ 2.5.2a was then used to align the remaining reads to the human transcriptome (build hg38). Xtail was used to count ribosome protected fragments, estimate translation efficiency, and perform statistical comparisons ${ }^{26}$.

\section{Polysome Profiling}

Polysome profiling was adapted from Gandin et. al.'s protocol and with direction and assistance from Dr. Alison Ashbrook in Dr. Charlie Rice's laboratory ${ }^{27}$. The day before cell collection, 7.5 million MDA-231 were plated in $15 \mathrm{~cm}$ plates (2 plates per experimental biological replicate) in normal DMEM media supplemented 551 with $10 \%$ FBS. Cells were plated to achieve approximately $80 \%$ confluency at 
552 collection time to optimize polysome content. Each plate was treated for 5 minutes 553 at $37^{\circ} \mathrm{C}$ with DMEM with $100 \mathrm{ug} / \mathrm{mL}$ cycloheximide. The plate was then transferred 554 to ice and the cycloheximide media was aspirated. Cells were washed twice with ice cold 1x PBS with $100 \mathrm{ug} / \mathrm{mL}$ cycloheximide. All PBS was then aspirated carefully and the $15 \mathrm{~cm}$ plate was flash frozen in liquid nitrogen. $425 \mathrm{uL}$ Lysis Buffer (5 mM Tris $\mathrm{HCl} \mathrm{pH} \mathrm{7.5,} 2.5 \mathrm{mM} \mathrm{MgCl}$, $1.5 \mathrm{mM} \mathrm{KCl}, 100 \mathrm{ug} / \mathrm{mL}$ cycloheximide, 2 mM DTT, $0.5 \%$ Triton $\mathrm{X}-100,0.5 \%$ sodium deoxycholate, and 100 units of SUPERase*In RNase Inhibitor (Invitrogen) 1x Protease Inhibitors EDTA-free) was then added to the plate and cells were scraped and transferred to an eppendorf tube on ice. Lysates were then spun at high speed at $4^{\circ} \mathrm{C}$ for 7 minutes to pellet nuclei. Supernatant was transferred to a new tube and the RNA concentration was measured using the Quant-iT Ribogreen assay (Life Technologies). 64ug RNA lysate was used for polysome fractionation. 10-50\% Sucrose gradients were prepared the day before ultracentrifugation. Ultracentrifuge polyallomer tubes (Beckman Coulter, Cat \#331372) were marked halfway and $\sim 5.5 \mathrm{~mL} \mathrm{10 \%} \mathrm{sucrose}$ polysome gradient buffer (20 mM Tris $\mathrm{HCl} \mathrm{pH} \mathrm{7.5,} 140 \mathrm{mM} \mathrm{KCl}, 5 \mathrm{mM} \mathrm{MgCl} 2,10 \%$ Sucrose, $100 \mathrm{ug} / \mathrm{mL}$ cycloheximide, $0.5 \mathrm{mM} \mathrm{DTT,} 20 \mathrm{U} / \mathrm{mL}$ SUPERase*In) was added with a $10 \mathrm{~mL}$ sterile syringe to $1 / 8$ inch above the line. $50 \%$ sucrose polysome gradient buffer $(20 \mathrm{mM}$ Tris $\mathrm{HCl} \mathrm{pH} \mathrm{7.5,} 140 \mathrm{mM} \mathrm{KCl}, 5 \mathrm{mM} \mathrm{MgCl} 2,50 \%$ Sucrose, $100 \mathrm{ug} / \mathrm{mL}$ cycloheximide, $0.5 \mathrm{mM}$ DTT, $20 \mathrm{U} / \mathrm{mL}$ SUPERase* ${ }^{\star n}$ ) was then underlain until the $10 \%$ sucrose layer was pushed above the marked line. The syringe was wiped with a Kimwipe prior to addition of $50 \%$ sucrose buffer to maintain separation between buffers. Black caps were added carefully to prevent the accumulation of bubbles in each ultracentrifuge tube. The Biocomp gradient master was then used at the following conditions: Long Cap 10\% - 50\% WV Step 1, 1:50 minutes, $80^{\circ}$ angle, 21 speed. Gradients were then sealed with parafilm and incubated at $4^{\circ} \mathrm{C}$ overnight. Gradients were then balanced to within $10 \mathrm{mg}$ of each other. Normalized cell lysates were added (500 uL volume) and spun in a SW41 ultracentrifuge rotor at 38,000 RPM for 2 hours at $4^{\circ} \mathrm{C} .60 \%$ sucrose was then used to fractionate spun lysates into $1 \mathrm{~mL}$ fractions and polysome peaks were measured with a Combi Flash UV-vis detector (Brandel) and TracerDAQ software. Polysome fractions were then pooled into appropriate groups: highly translated (Higher than 3 ribosomes, past the $1^{\text {st }}$ peak), and lowly translated (1-2 ribosomes and $80 \mathrm{~s}$ ) based on A280 UV peaks. The pooled fractions were then incubated with 3:1 Trizol LS Reagent, vortexed thoroughly, and incubated at RT for 5 minutes. RNA was then extracted following the instructions of the Direct-zol Miniprep Ki (Zymo Research), and eluted in $50 \mathrm{uL}$. RNA was quantified and normalized for input into either tRNACapture-seq qPCR with 5S, tRNA ${ }^{l l e}{ }_{G A U}$, or tRNA ${ }^{\text {lle }}$ UAU probes (250 ng) or as Input into the QuantSeq 3' mRNA-Seq Library Prep Kit (Lexogen) kit. 500 ng RNA was used as input and samples were processed according to QuantSeq (Lexogen) instructions. A pooled library was compiled using manufacturer's primers and $10 \mathrm{nM}$ Pool was quality checked with Tapestation and sequenced on the NextSeq High Output 75 Single Read at the Rockefeller University Genomics Resource Center.

For analysis, reads were mapped to the human transcriptome using STAR (v2.5.2a) with genome build hg38 and the number of reads for each gene was 
tabulated using featureCounts (v1.6.1). The Bioconductor package DESeq2 was then used to compare the fractions in control and tRNA ${ }^{\mathrm{lle}}{ }_{\text {GAU }} /$ tRNA $^{\mathrm{lle}}$ UAU modulated 600 samples.

601

602

\section{Proteomics} protease inhibitors (Roche). 50 ug lysate was used for label free quantification at the Rockefeller University Proteomics Core Facility. Maxquant software was utilized to compare three replicates per experimental group. Label free quantitation (LFQ) was used to compare the same peptide/protein between experimental groups ( $n=3$ samples per group), which relies on normalization and strict filter criteria determined by the Proteomics Core. Student's t-test difference and student's t-test was then used to analyze the data.

\section{Immunofluorescence}

Paraffin embedded histology slides from metastatic nodules were used. Slides underwent deparaffination via 5 minute incubations in xylene 2x, 100\% Ethanol, 2x, 70\% Ethanol, 50\% Ethanol, and subsequently 1x PBS. Antigen retrieval was performed with $1 \times$ Citrate Buffer $\mathrm{pH} 6.0$ in a microwave for 20 minutes. Slides were cooled to room temperature, then tissue regions were isolated with a PAP pen. Slides were then blocked with 10\% Goat Serum (Sigma Aldrich) for 30 minutes at room temperature. Primary antibodies were incubated overnight at $4^{\circ} \mathrm{C}$ in a moist chamber (Vimentin V9 mouse (Abcam ab8069) 1:50), or for 2 hours at room temperature (Ki67 (Abcam ab927420) 1:200). Slides were washed with 0.5\% Tween 20 PBS then incubated with secondary antibody for 1 hour at RT (1:200). Slides were then stained with DAPI for 5 minutes, and sealed with Prolong Gold anti-fade solution (Thermo Fisher). Fluorescent intensity was measured on an Inverted TCS SP8 laser scanning confocal microscope (Leica) at the Bioimaging Resource Center at Rockefeller University and quantified by number of positive cells per field of view. Quantification was performed blind.

\section{In Vitro Growth Assays}

Cells at similar confluencies were resuspended in new DMEM media and counted with a hemacytometer. Cells were then seeded in equal numbers (100K for stress conditions, $50 \mathrm{~K}$ for normal in vitro conditions) in 6 well plates in triplicate. Cells were then counted at the endpoint day with a hemacytometer. Each experiment was conducted three times. Cells treated with 200 uM H202 were counted on Day 3. Cells exposed to $0.5 \%$ hypoxia in an InvivO ${ }^{2}$ chamber (Baker Ruskinn) were quantified on Day 3. Growth assays in normal in vitro conditions were quantified on Day 5.

\section{Western Blot}


644 a 4-12\% Bis-Tris or 3-8\% Tris Acetate gel (Invitrogen), and then transferred at 300 $645 \mathrm{~mA}$ for one hour in 15\% methanol 1x Transfer Buffer on a methanol activated 646 PVDF membrane. Membranes were then stained with Ponceau and blocked for one hour in Odyssey ${ }^{\circledR}$ Blocking Buffer. Primary antibody incubations occurred overnight at $4^{\circ} \mathrm{C}$ on a rocker at the following concentrations: alpha tubulin 1:1000, (Proteintech) SMNDC1 1:500 (Proteintech). Membranes were then washed with $0.05 \%$ Tween 20 PBS three time and incubated with mouse or rabbit fluorescent IRDye ${ }^{\circledR}$ conjugated secondary antibodies 1:20,000 (LI-COR Biosciences) for one hour. Membranes were subsequently washed three times and imaged and quantified using the Odyssey ${ }^{\circledR}$ Sa Infrared Imaging System at the Rockefeller University Center for High Throughput Screening. Quantification was done using the Image Studio Lite ${ }^{\mathrm{TM}}$ software.

\section{Codon Reporters}

Wildtype or codon mutant SMNDC1 (all AUA codons changed to AUC codons) coding sequence gene blocks were designed with Nhel \& Xhol restriction sites and a N-terminal flag tag and ordered from IDT. SMDNC1 gene blocks were cloned into the psiCheck 2 vector. The firefly luciferase was removed and replaced with a renilla luciferase with only $A U U$ encoding isoleucines via restriction cutting with PspOMI \& Xbal. This adapted SMNDC1 reporter was transfected with $2.5 \mathrm{ug}$ plasmid and $10 \mathrm{uL}$ Lipofectamine 2000 (Thermo Fisher) in triplicate in MDA-MB231 cells with modulated tRNA ${ }^{\text {lle }}$ levels. Cells were lysed after 24 hours and protein was extracted with RIPA buffer with $1 \mathrm{x}$ protease inhibitors EDTA-free (Roche). Protein expression was measured through LICOR Western blotting as described above.

\section{RSCU and Pathway Enrichment Analyses}

Gene filtering: The Homo sapiens GRCh38 CDS sequences were downloaded from the Ensembl database. To avoid multiple splice variants from the same gene affecting downstream analysis, principle splice isoforms were filtered using annotations from the APPRIS database and a custom Python script. For genes with multiple annotated isoforms, the transcript with the highest score was chosen as the representative.

RSCU calculation: To calculate the relative synonymous codon usage (RSCU) for a given codon in each gene, we first calculated the total abundances of each codon across our entire filtered CDS dataset to determine the empiric distribution of synonymous codon usage for each amino acid. For each gene, the RSCU score was calculated as: [Observed_Codon_Usage - Expected_Codon_Usage] / Expected_codon_usage. The expected codon usage was defined as Observed_Amino_Acid_Usage * $\mathrm{Pr}$ (Codon_Usage | Amino_Acid) where the probability mass function was determined using the empiric codon distribution described above. For genes/transcripts in which a given amino acid appeared zero times, the RSCU score was set to 0 .

calculated above. Mutual information analyses to detect significantly over689 represented and under-represented pathways in discrete bins were performed 
690 using the iPAGE mutual information framework with pathway annotations built from 691 the Reactome database ${ }^{28}$. Heatmaps were generated with iPAGE. To determine 692 pathways that were most likely to be divergently modulated by AUA or AUC 693 over/under-expression, we additionally filtered the output to include pathway 694 enrichments/depletions present in both AUC and AUA analyses with p-values less 695 than 10E-3 in the highest RSCU bin. Heatmaps were generated using Python 696 software and the seaborn package.

697 Databases/Sites/Software used:

698 Ensembl: https://useast.ensembl.org/index.html

699 APPRIS: http://appris-tools.org/\#/

700 iPAGE: https://tavazoielab.c2b2.columbia.edu/iPAGE/

701 Reactome: https://reactome.org

702 Python 3.6.0: https://www.python.org

703 Pandas: https://pandas.pydata.org

704 Seaborn: https://seaborn.pydata.org

\section{Statistical analysis}

Results are presented in dot-plot with dots representing individual values and barcharts depicting average values with standard error of the mean ( \pm s.e.m.). The number of samples for each group was chosen based on the expected levels of variation and consistency. FISH quantification was performed in a blinded fashion. Unless otherwise stated, statistical significance was assessed by a two-tailed Student's t-test with $P$-value $<0.05$ being considered statistically significant.

\section{Data availability}

Experimental data will be available from the corresponding author upon request. Sequencing data will be made available in public databases.

\section{Ethical regulations}

All animal experiments were performed under supervision and approval of the Institutional Animal Care and Use Committee (IACUC) at the Rockefeller University.

Acknowledgements We thank members of the Tavazoie laboratory and Hani Zaher for thoughtful comments on previous versions of the manuscript. We thank Alison Ashbrook and Charlie Rice's laboratory for technical assistance with polysome profiling. We also thank Rockefeller University resource centers: Alison North and staff at the Bio-Imaging resource facility, Connie Zhao from the genomics resource center, Soren Heissel and Henrik Molina from the proteomics resource center, and Vaughn Francis from the Comparative bioscience center and veterinary staff for animal husbandry and care. L.N and M.C.P. were supported by a Medical Scientist Training Program grant from the National Institute of General Medical Sciences of the National Institutes of Health under award number T32GM007739 to the Weill Cornell/Rockefeller/Sloan Kettering Tri-Institutional

734 S.F.T. is an HHMI Faculty Scholar, and was supported by the Breast Cancer 
735 Research Foundation award, the Reem-Kayden award, and by $\mathrm{NCl}$ grant

736 R01CA215491. S.F.T. and the Tavazoie lab were supported by the Black Family 737 and the Black Family Metastasis Research Center.

Author Contributions L.N., H.G. and S.F.T. designed the experiments. L.N, N.M., 841 and M.C.P. performed the experiments. H.G. and H.A. performed ChIP-Seq, 842 TGIRT, Ribosomal Profiling, Polysome Profiling sequencing, and ribosomal 843 dwelling time analyses. D.H. performed iPAGE codon analyses. L.N. and S.F.T. 844 wrote the paper with input from the co-authors.

845

\section{References}

1 Ogle, J. M., Carter, A. P. \& Ramakrishnan, V. Insights into the decoding mechanism from recent ribosome structures. Trends Biochem Sci 28, 259-266, doi:10.1016/S0968-0004(03)00066-5 (2003).

2 Schmeing, T. M. \& Ramakrishnan, V. What recent ribosome structures have revealed about the mechanism of translation. Nature 461, 1234-1242, doi:10.1038/nature08403 (2009).

3 Pavon-Eternod, M. et al. tRNA over-expression in breast cancer and functional consequences. Nucleic Acids Res 37, 7268-7280, doi:10.1093/nar/gkp787 (2009).

4 Goodarzi, H. et al. Modulated Expression of Specific tRNAs Drives Gene Expression and Cancer Progression. Cell 165, 1416-1427, doi:10.1016/j.cell.2016.05.046 (2016).

5 Gingold, H. et al. A dual program for translation regulation in cellular proliferation and differentiation. Cell 158, 1281-1292, doi:10.1016/j.cell.2014.08.011 (2014).

6 Robichaud, N., Sonenberg, N., Ruggero, D. \& Schneider, R. J. Translational Control in Cancer. Cold Spring Harb Perspect Biol 11, doi:10.1101/cshperspect.a032896 (2019).

7 Vo, M. N. et al. ANKRD16 prevents neuron loss caused by an editing-defective tRNA synthetase. Nature 557, 510-515, doi:10.1038/s41586-018-0137-8 (2018).

8 Knott, S. R. V. et al. Asparagine bioavailability governs metastasis in a model of breast cancer. Nature 554, 378-381, doi:10.1038/nature25465 (2018).

9 Loayza-Puch, F. et al. Tumour-specific proline vulnerability uncovered by differential ribosome codon reading. Nature 530, 490-494, doi:10.1038/nature16982 (2016).

10 Minn, A. J. et al. Genes that mediate breast cancer metastasis to lung. Nature 436, 518-524, doi:10.1038/nature03799 (2005).

11 Goodarzi, H., Elemento, 0. \& Tavazoie, S. Revealing global regulatory perturbations across human cancers. Mol Cell 36, 900-911, doi:10.1016/j.molcel.2009.11.016 (2009). 


\begin{tabular}{|c|c|c|}
\hline 880 & \multirow[t]{3}{*}{12} & y, N. J. \& Ingolia, N. T. Transcriptome-wide measurement of translation \\
\hline 881 & & ribosome profiling. $\quad$ Methods $\quad \mathbf{1 2 6}$, \\
\hline 882 & & doi:10.1016/j.ymeth.2017.05.028 (2017). \\
\hline 883 & \multirow[t]{3}{*}{13} & Gill, J. G., Piskounova, E. \& Morrison, S. J. Cancer, Oxidative Stress, and \\
\hline 884 & & Metastasis. Cold Spring Harb Symp Quant Biol 81, 163-175, \\
\hline 85 & & doi:10.1101/sqb.2016.81.030791 (2016). \\
\hline 886 & 14 & Loo, J. M. et al. Extracellular metabolic energetics can promote cancer \\
\hline 37 & & 3-406, doi:10.1016/j.cell.2014.12.018 (2015). \\
\hline 388 & \multirow[t]{3}{*}{15} & Nguyen, A., Yoshida, M., Goodarzi, H. \& Tavazoie, S. F. Highly variable cancer \\
\hline 39 & & ubpopulations that exhibit enhanced transcriptome variability \\
\hline 890 & & etastatic fitness. Nat Commun 7, 11246, doi:10.1038/ncomms11246 (2016). \\
\hline 1 & 6 & iskounova, E. et al. Oxidative stress inhibits distant metastasis by human \\
\hline 2 & & 1oma cells. Nature 527, 186-191, doi:10.1038/nature15726 (2015). \\
\hline 93 & \multirow[t]{3}{*}{17} & Katibah, G. E. et al. Broad and adaptable RNA structure recognition by the \\
\hline 4 & & interferon-induced tetratricopeptide repeat protein IFIT5. Proc Natl \\
\hline 95 & & Acad Sci U S A 111, 12025-12030, doi:10.1073/pnas.1412842111 (2014). \\
\hline 6 & \multirow[t]{3}{*}{18} & Mohr, S. et al. Thermostable group II intron reverse transcriptase fusion \\
\hline 97 & & use in cDNA synthesis \\
\hline & & ncing. $R N A$ 19, 958-970, doi:10.1261/rna.039743.113 (2013). \\
\hline 99 & 19 & Goodarzi, H. RiboLog, <github.com/Goodarzilab/Ribolog> (2019). \\
\hline 00 & \multirow[t]{3}{*}{20} & Nguyen, A. et al. PKLR promotes colorectal cancer liver colonization through \\
\hline 1 & & induction of glutathione synthesis. $J$ Clin Invest 126, 681-694, \\
\hline 02 & & $0.1172 / J C I 83587$ (2016). \\
\hline 03 & \multirow[t]{4}{*}{21} & Chaudhuri, A. D., Yelamanchili, S. V. \& Fox, H. S. Combined fluorescen \\
\hline & & zation for detection of microRNAs and imr \\
\hline 05 & & cell-type markers. Front Cell Neurosci 7, 160, doi:10.3389/fncel.2013.00160 \\
\hline 6 & & 3). \\
\hline 07 & \multirow[t]{2}{*}{22} & tivirus-del \\
\hline 78 & & \\
\hline 09 & \multirow[t]{2}{*}{23} & ryant, D. M. et al. A molecular network for de novo generation of the apical \\
\hline 9 & & and lumen. Nat Cell Biol 12, 1035-10 \\
\hline 91 & \multirow[t]{3}{*}{24} & Chen, B. et al. Dynamic imaging of genomic loci in living human cells by an \\
\hline 912 & & CRISPR/Cas \\
\hline 01 & & doi:10.1016/j.cell.2013.12.001 (2013). \\
\hline 912 & \multirow[t]{3}{*}{25} & Yeo, N. C. et al. An enhanced CRISPR repressor for targeted mammalian gene \\
\hline & & regulation. Nat Methods 15, 611-616, doi:10.1038/s41592-018-0048-5 \\
\hline 91 & & \\
\hline 01" & \multirow[t]{3}{*}{26} & Q., Liu, Y. \& Yang, X. Genome-wide assessment of differe \\
\hline & & translations with ribosome profiling data. Nat Commun $\mathbf{7}, 1119$ \\
\hline & & doi:10.1038/ncomms11194 (2016). \\
\hline 920 & 27 & Gandin, V. et al. nanoCAGE reveals 5' UTR features that define specific modes \\
\hline & & slation of functionally related MTOR-sensitive mRNAs. Genome Res 2 \\
\hline 922 & & 636-648, doi:10.1101/gr.197566.115 (2016). \\
\hline 923 & 28 & Goodarzi, H., Hottes, A. K. \& Tavazoie, S. Global discovery of adap \\
\hline & & tions. Nat Methods 6, 581-583, doi:10.1038/nmeth.1352 (2009). \\
\hline & & \\
\hline
\end{tabular}




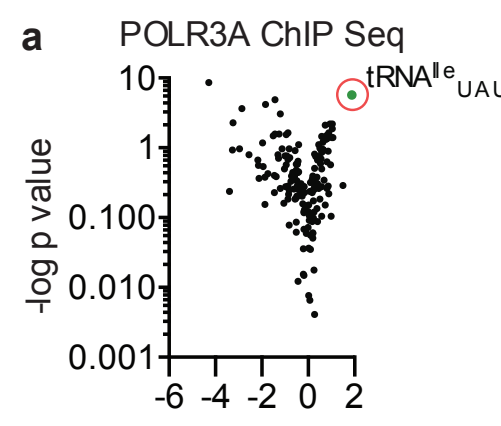

log2 Fold Change

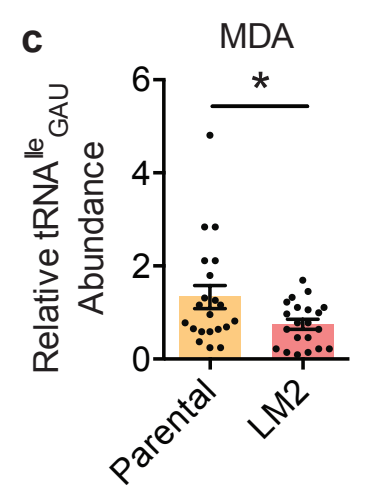

d
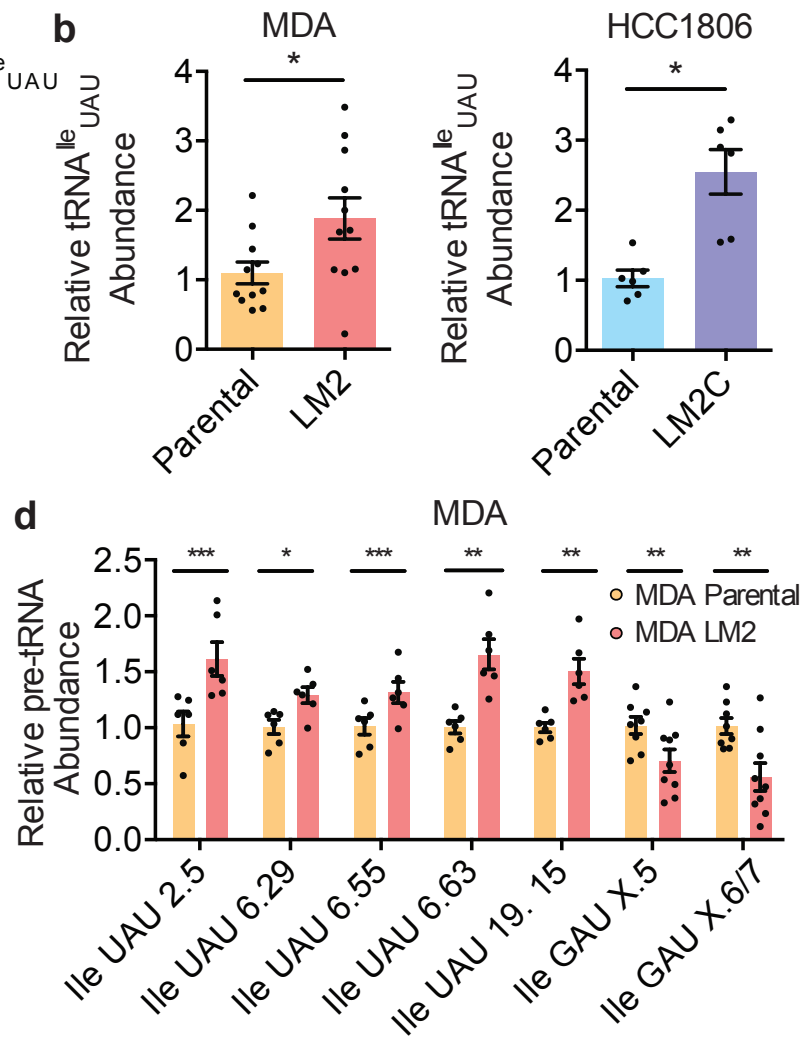

Genomic tRNA Loci
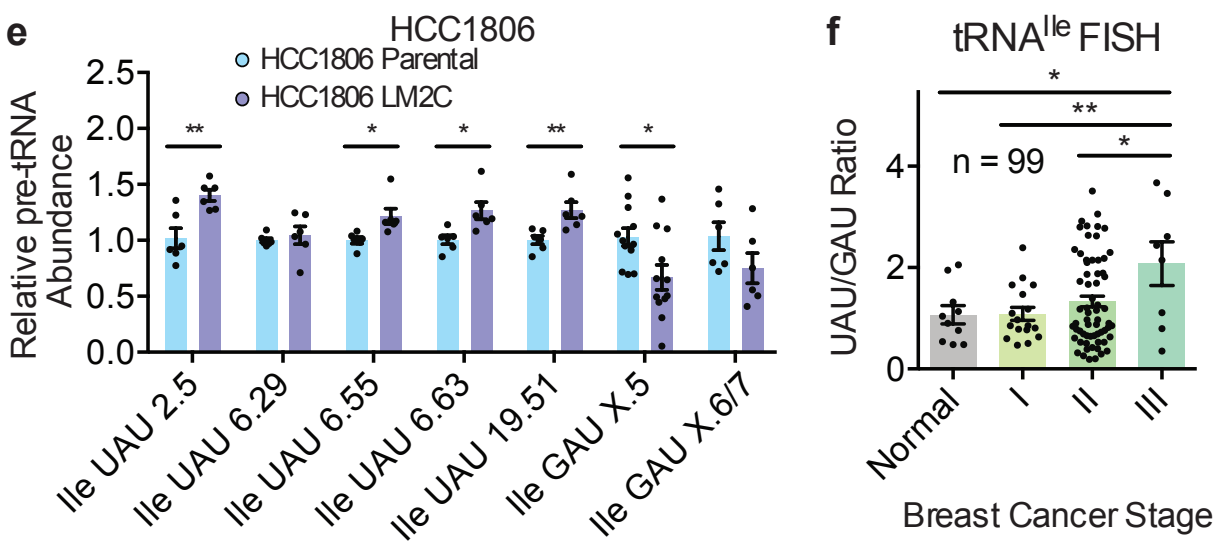

Genomic tRNA Loci

926

927

928

929

930

931

932

933
Figure 1 - Isoleucine isoacceptors are differentially modulated in isogenic poorly and highly metastatic breast cancer pairs.

(a) Volcano plot representing log2 fold change vs. -log $p$ value of POL3RA ChIP sequencing analysis of MDA-LM2 cells vs. MDA-MB-231 Parental cells.

(b) tRNA ${ }^{\text {lle }}$ UAU quantification by specific tRNA ${ }^{\text {lle }}$ UAU probe RT-qPCR normalized to $18 S$ probes of highly metastatic LM2 lines relative to their parental MDA-MB-231 and HCC1806 cell lines. 
934 (c) tRNA ${ }^{1 l e}$ GAU quantification by specific tRNA ${ }^{11 \mathrm{e}}$ GAU probe RT-qPCR normalized to $93518 \mathrm{~S}$ probes of highly metastatic LM2 lines relative to the parental MDA-MB-231 936 cell line.

937 (d,e) Relative pre-tRNA abundance of tRNA ${ }^{l l e}$ UAU and tRNA ${ }^{\text {lle }}$ GAU across multiple 938 primers covering distinct genetic loci using RT-qPCR of MDA-LM2 vs. MDA-MB939231 (d) \& HCC1806-LM2C vs. HCC1806 Parental cells (e).

940 (f) Relative tRNA ${ }^{l l e}$ UAU/tRNA ${ }^{l l e}{ }_{G A U}$ ratios quantified by fluorescent intensity 941 normalized to DAPI of breast tissue microarrays, stratified by normal tissue or 942 breast cancer stage I \& II, III, measured by FISH with LNA targeting tRNA ${ }^{\text {Ile }}$ UAU or 943 tRNA $^{l l e}{ }_{\text {GAU }}$. Two-sided un-paired student's t-tests performed, $p$-values $p<0.05$, $944 \mathrm{p}<0.01, \mathrm{p}<0.001$ represented as ${ }^{*},{ }^{* *},{ }^{* * *}$, respectively.

945

946 

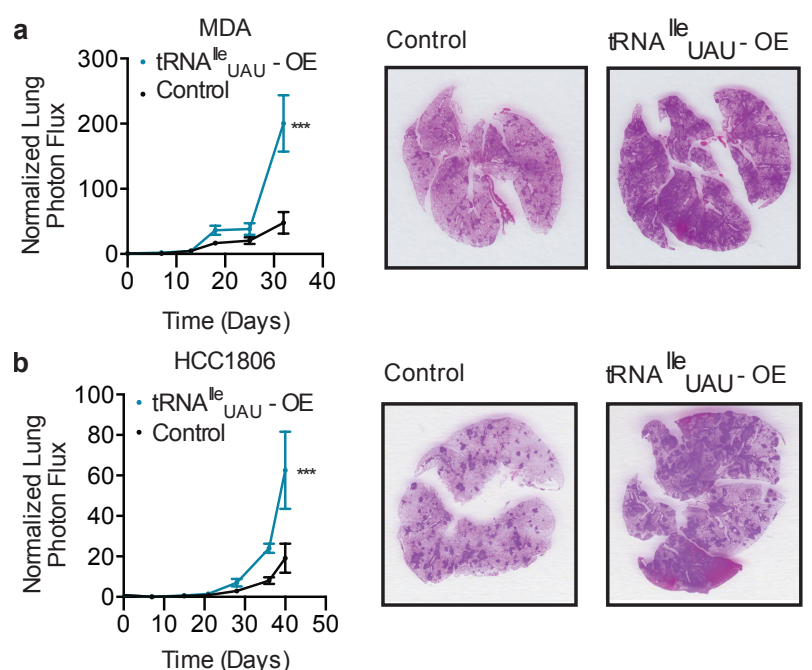

c MDA LM2 CRISPRV2
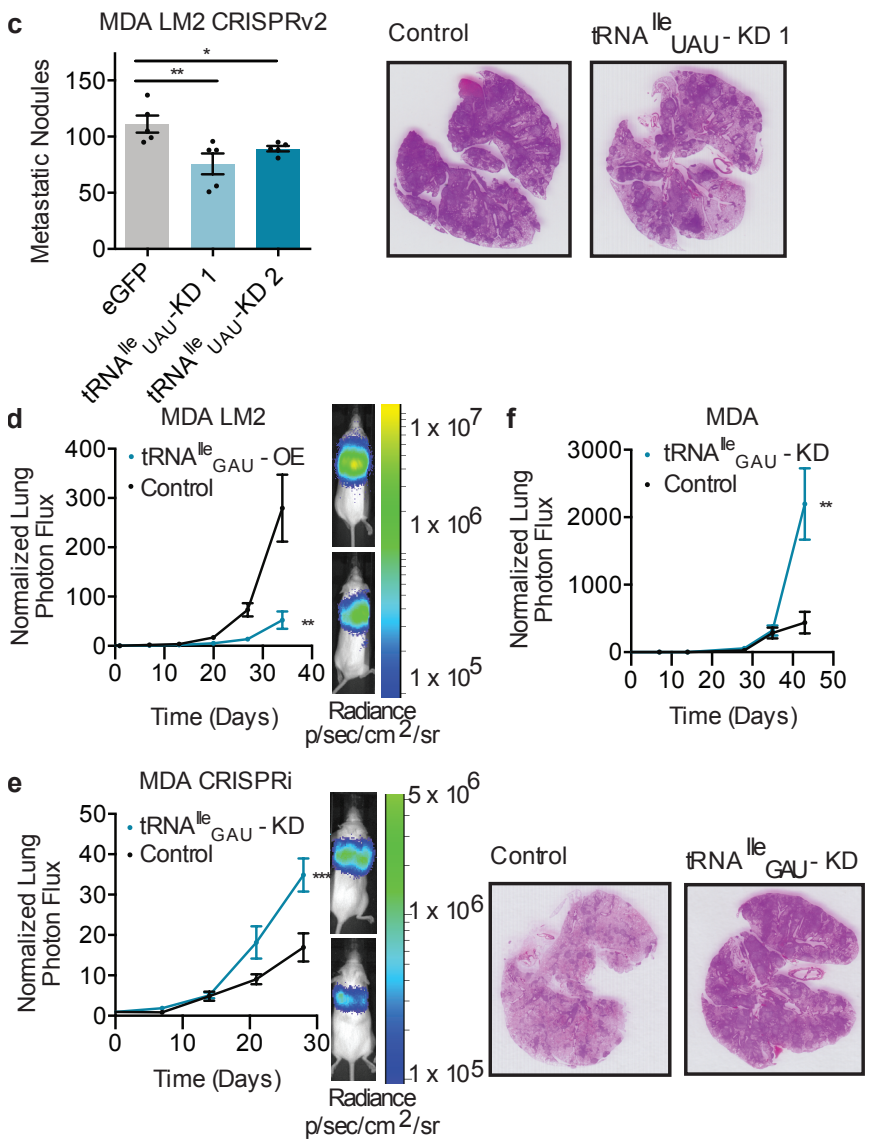

947 Figure 2 - tRNA ${ }^{\text {Ile }}$ UAU promotes \& tRNA $^{\text {lle }}$ GAU suppresses metastatic 948 colonization .

949 (a-b) Bioluminescent imaging post tail vein injection of $1 \times 10^{5}$ of MDA Parental (a) 950 or $1.5 \times 10^{5}$ HCC1806 (b) cells overexpressing tRNA ${ }^{1 \mathrm{e}} \mathrm{UAU}$ or control with 951 representative lung histology stained with $\mathrm{H} \& \mathrm{E} ; \mathrm{n}=5$ in each cohort.

952 (c) Quantification of lung metastatic nodules post extraction after tail vein injection 953 of $5 \times 10^{4}$ LM2 CRISPR cells guides targeting eGFP or tRNA ${ }^{\text {lle }}$ UAU, with 954 representative histology for control \& RRNA $^{\| l e}$ UAU guide $1 ; n=5$ in each cohort. 
955 (d) Bioluminescence imaging after tail vein injection of $1 \times 10^{5}$ of MDA LM2 cells 956 overexpressing tRNA ${ }^{\text {lle }}$ GAU or control with representative images; luminescence 957 expressed as Radiance $\mathrm{p} / \mathrm{sec} / \mathrm{cm}^{2} / \mathrm{sr} ; \mathrm{n}=5$ in each cohort.

958 (e,f) Same as (d) with MDA Parental CRISPRi cells with guides targeting either 959 control or tRNA ${ }^{1 \mathrm{le}}{ }_{\text {GAU }}(\mathrm{e})$ or shRNA targeting control or tRNA ${ }^{\mathrm{lle}}{ }_{\text {GAU }}$ with representing 960 H\&E lung histology (f). Statistics utilized include 2-way ANOVA for imaging and 961 two-sided unpaired student's t-test for nodule quantification, $p$-values $p<0.05$, 962

963

964

965

966

967

968

969 


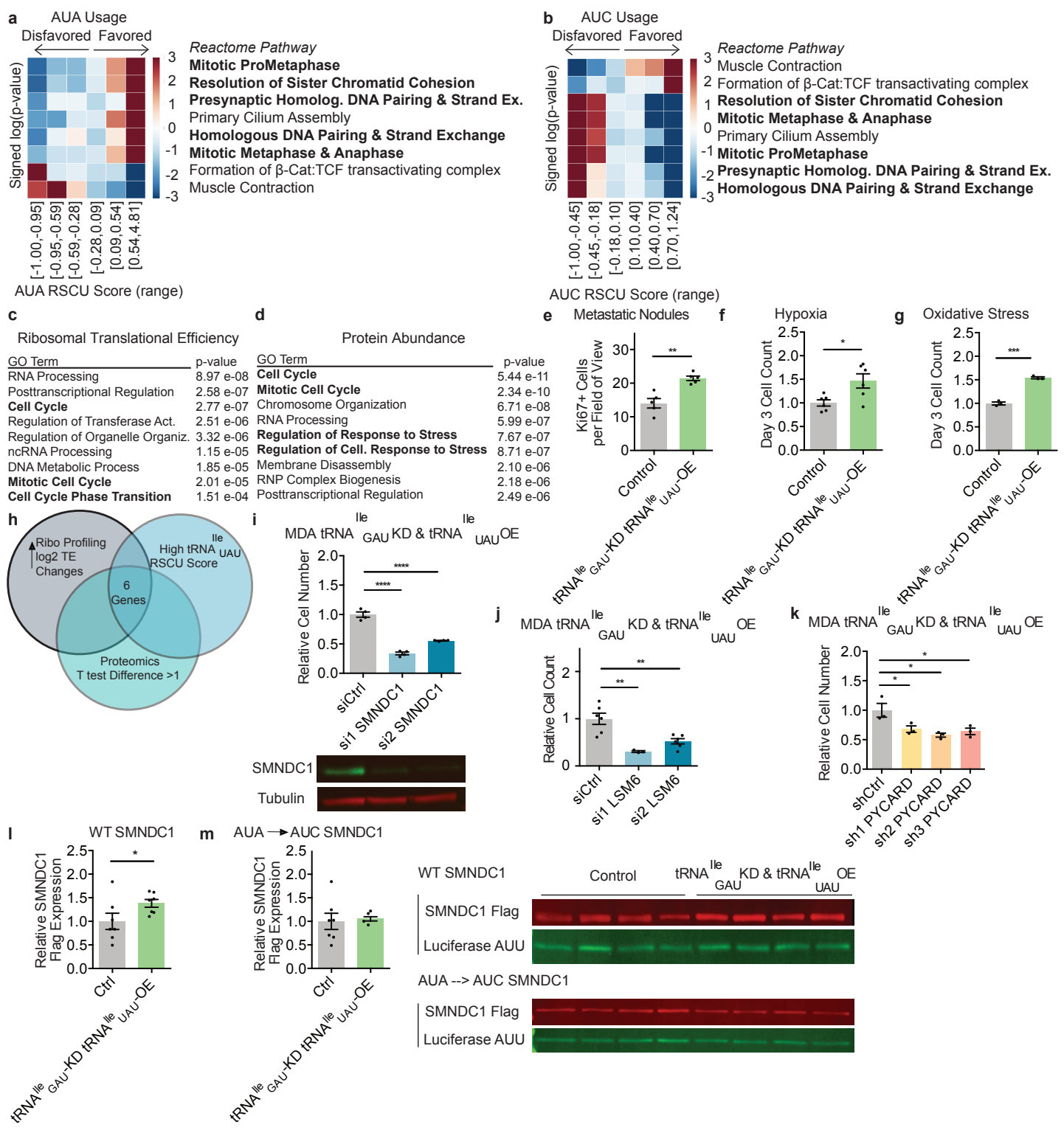

Figure 3 - Cell Cycle and Response to Stress gene expression and

973 (a,b) Reactome pathways significantly enriched in AUA (a) or AUC (b) by relative 974 synonymous codon usage (RSCU) using iPAGE.

975 (c,d) GO function terms for positive and significant TE changes from ribosomal 976 profiling (c) or label free quantification by mass spectrometry (d) in tRNA ${ }^{\text {lle }}$ GAU 977 depletion and tRNA ${ }^{\text {lle }}$ UAU overexpression cells versus control.

978 (e) Quantification of Ki67 immunofluorescence staining in MDA MB 231 tRNA ${ }^{\text {lle }}$ GAU 979 depletion and tRNA ${ }^{\text {Ile }}$ UAU Overexpression cells versus control.

980 (f,g) Relative cell counts of MDA MB 231 control \& tRNA ${ }^{\text {lle }}$ GAU depletion tRNA ${ }^{\text {lle }}$ UAU 981 overexpression cells exposed to $0.5 \%$ hypoxia (f) or treated with $200 \mathrm{uM} \mathrm{H}_{2} \mathrm{O}_{2}(\mathrm{~g})$ 982 for 3 days. 
983 (h) Venn Diagram of overlapping datasets to identify downstream effectors 984 includes high RSCU tRNA ${ }^{\text {lle }}$ UAU score (top 50\%), and genes with significantly 985 positive changes in TE and proteomics in both MDA LM2 vs. MDA Parental cells 986 and tRNA ${ }^{l l e}$ GAU depletion tRNA ${ }^{\text {lle }}$ UAU Overexpression cells vs. control.

987 (i) Relative cell count of MDA tRNA ${ }^{\text {lle }}$ GAU depletion tRNA ${ }^{\text {lle }}$ UAU Overexpression cells 988 treated with control or SMNDC1 siRNA in 0.5\% hypoxia for 2 days $(d)$. Western 989 performed on siRNA cells on day 3.

990 (j) Relative cell counts of MDA tRNA ${ }^{\text {lle }}$ GAU depletion tRNA ${ }^{l l e}$ UAU overexpression 991 cells treated with control or LSM6 siRNA for 3 days.

992 (k) Relative cell counts of MDA tRNA ${ }^{\text {lle }}$ GAU depletion tRNA ${ }^{l l e}$ UAU overexpression 993 cells transduced with shRNA targeting either control or PYCARD for 3 days.

$994(\mathrm{I}, \mathrm{m})$ LICOR Western quantification of either Flag tagged wildtype (I) or all AUA to 995 AUC codons (m) SMNDC1 expression relative to reporter control luciferase (all lle 996 AUU) 24 hours post transfection in either MDA control or tRNA ${ }^{\text {lle }}$ GAU depletion 997 tRNA ${ }^{\text {Ile }}$ UAU Overexpression cells. Representative images below. Statistics utilized 998 include two-sided un-paired student's's t-tests performed, p-values * ${ }^{* *},{ }^{* * * *}$ 999 indicated as $p<0.05, p<0.01$, and $p<0.0001$, respectively. 


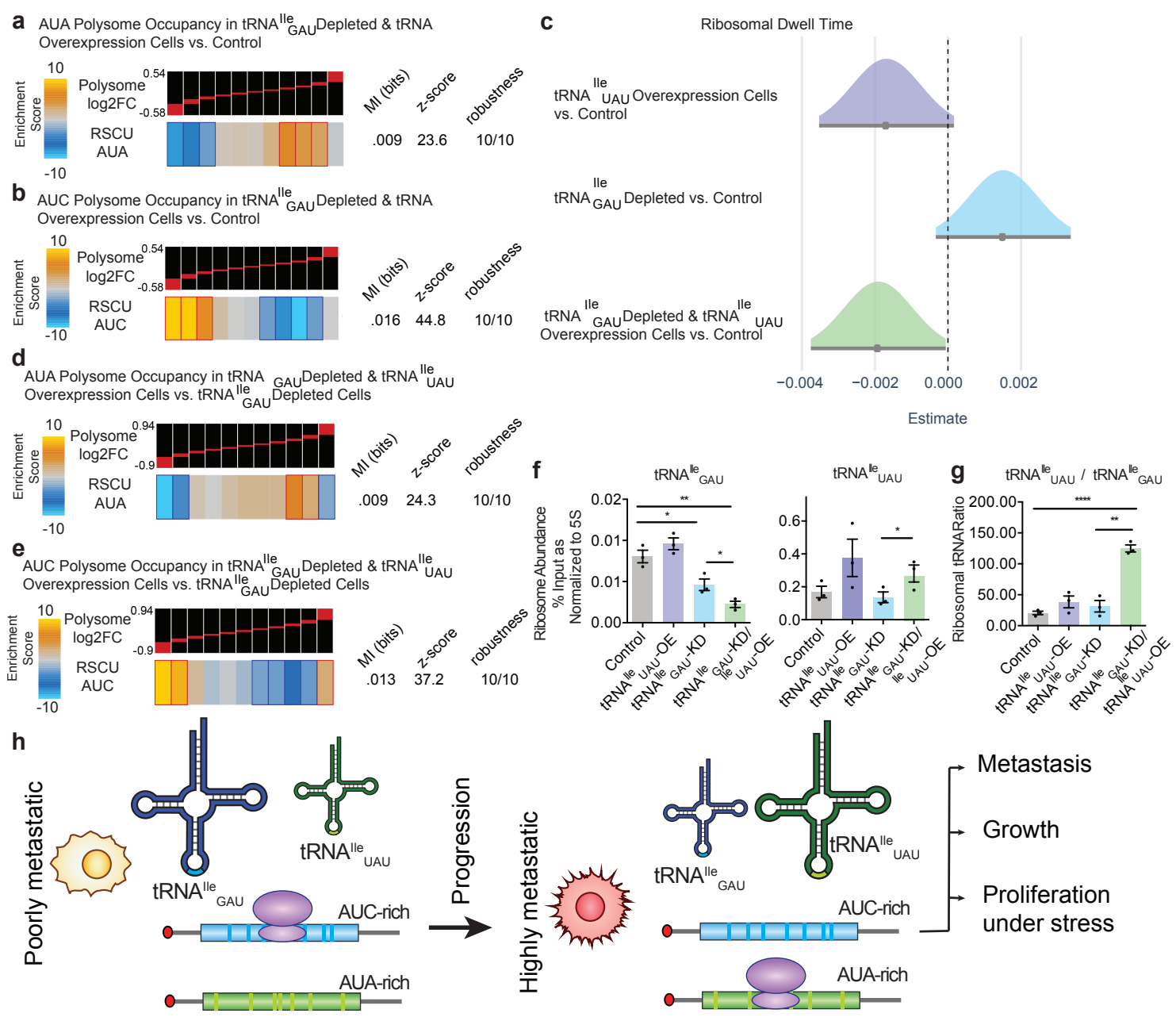

Figure 4 - Translational efficiency of AUA enriched transcripts is dependent on tRNA ${ }^{\text {lle }}$ GAU abundance.

(a) Genes with a high abundance of AUA codons (using RSCU scores) were significantly enriched among genes upregulated in polysomes (corrected for their transcript changes) in tRNA ${ }^{\text {lle }}$ GAU depleted tRNA ${ }^{l l e}$ UAU overexpression cells versus control MDA-MB-231 cells. The statistical significance of these enrichments was assessed using mutual-information calculations and associated Z score (based on randomized input vectors) and robustness scores (based on jackknifing tests). The heatmap was generated using the -log of the hypergeometric $p$-value for enrichment and log of $p$-value for depletion (collectively termed the enrichment score). The red and dark-blue borders indicate the statistical significance of the calculated hypergeometric p-values (for details, see Goodarzi et al., 2009) ${ }^{11}$. (b) Same as (a) except analyzed for AUC codon enrichment, showing significantly depletion among genes upregulated in polysomes (corrected for their transcript changes) in tRNA ${ }^{l l e}$ GAU depleted tRNA ${ }^{l l e}$ UAU overexpression cells versus control MDA-MB-231 cells.

1025 (c) Ribosomal AUA codon dwelling times as estimated by CELP bias coefficients (higher bias coefficient indicates longer dwelling time). Univariate regression coefficients estimating the effects of tRNAlle modulated MDA cells. A 95\% 
1026 confidence interval excluding zero (not overlapping the vertical line $x=0$ ) means 1027 that the tested effect was significant at $\alpha=0.05(p<0.05)$.

1028 (d) Hypergeometric distribution shown as a z- score of AUA codon enrichment of 1029 polysome transcripts represented as log2 fold change of tRNA ${ }^{\text {lle }}$ GAU depleted 1030 tRNA ${ }^{\text {lle }}$ UAU overexpression cells versus tRNA ${ }^{l l e}$ GAU depleted MDA MB 231 cells, 1031 stratified in bins of 10 , increased log2 fold change from left to right. AUA codon 1032 representation visualized as a value ranging from -10 to 10 relative to the average.

1033 (e) Same as (d) except analyzed for AUC codon enrichment.

1034 (f) Ribosome abundance of tRNA ${ }^{\text {lle }}$ quantified by specific tRNA ${ }^{\text {lle }}$ GAU (left) and 1035 tRNA $^{\text {Ile }}$ UAU (right) probes. RT-qPCR normalized to $5 S$ probes of tRNA ${ }^{\text {lle }}$ modulated 1036 MDA cells from polysome fractions, measured as \% Input.

1037 (g) Ribosomal ratio of tRNA ${ }^{\text {lle }}$ UAU/ tRNA ${ }_{\text {GAU }}$ abundance quantified by specific 1038 tRNA $^{\text {lle }}$ GAU probe and tRNA ${ }^{\text {lle }}$ UAU probe RT-qPCR normalized to $5 S$ probes of 1039 tRNA ${ }^{\text {lle }}$ modulated MDA cells from polysome fractions, measured as \% Input.

1040 (h) Model depicting how tRNA ${ }^{\text {lle }}$ abundance shifts alter translational dynamics and 1041 metastatic phenotypes. Two-sided un-paired student's's t-tests performed, pvalues represented as * ${ }^{* *}$ as $p<0.05, p<0.01$ respectively. 

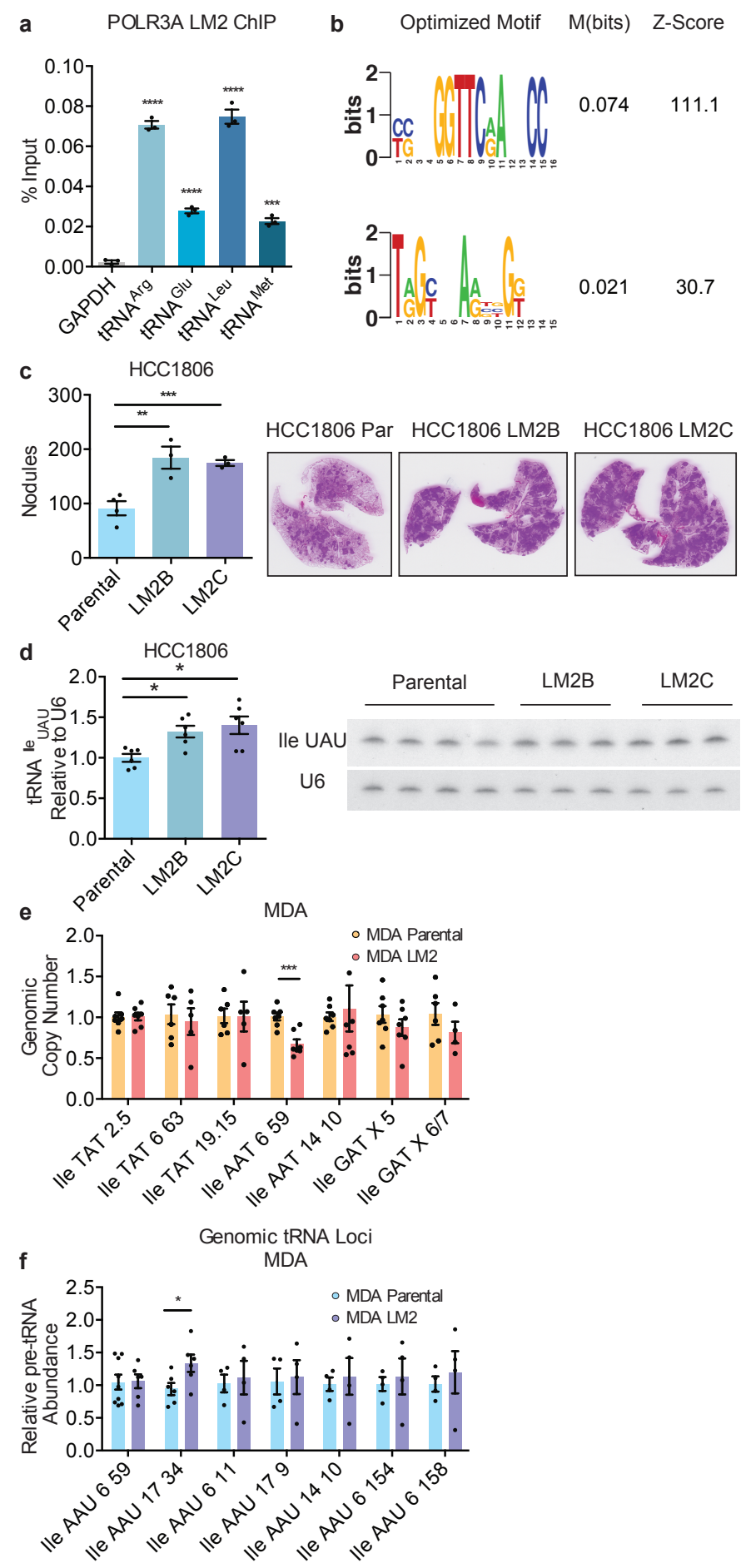

Genomic tRNA Loci

1044 Supplementary Figure 1 - POLR3A ChIP sequencing reveals differential 1045 occupancy in isogenic poorly and highly metastatic breast cancer pairs.

1046 (a) tRNA genomic loci abundance measured as percent (\%) input using RT-qPCR 1047 of POLR3A IP cDNA with GAPDH as a negative control. 
1048 (b) Motif analysis of POLR3A IP normalized to Input sequences using FIRE 1049 analysis.

(c) Quantification of lung metastatic nodules post extraction after tail vein injection of $1.5 \times 10^{5} \mathrm{HCC} 1806$ Parental or highly metastatic derivatives LM2B or LM2C, with representative histology; $n=3-4$ in each cohort.

(d) Northern blot quantification of tRNA ${ }^{\text {lle }}$ UAU relative to U6 of two independently derived LM2 lines relative to $\mathrm{HCC} 1806$ Parental cells with representative blot.

(e) Relative genomic copy number of tRNA lle loci, quantified by RT-qPCR.

(f) Relative pre-tRNA abundance of tRNA ${ }^{\text {lle }}$ AAU across multiple primers covering distinct genetic loci using RT-qPCR of MDA LM2 vs. MDA-MB-231. Two-sided unpaired student's t-tests performed, p-values represented ${ }^{*},{ }^{* *},{ }^{* * *},{ }^{* * *}$ as $p<0.05$, 1060

1061 $p<0.01, p<0.001, p 0.0001$, respectively.
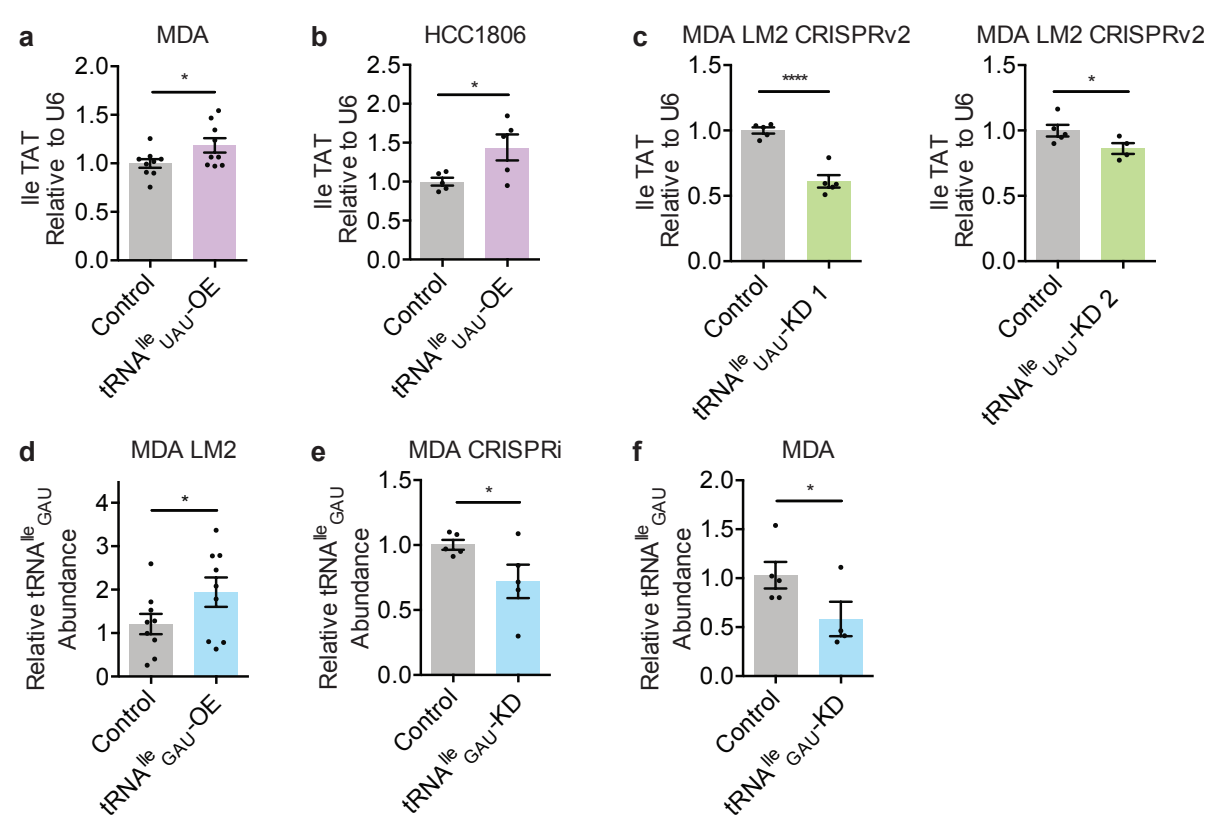

1062

1063

Supplementary Figure 2 - tRNA ${ }^{l l e}$ UAU and tRNA ${ }^{l l e}$ GAU levels can be manipulated exogenously.

1064

$(a, b)$ Northern blot quantification of tRNA ${ }^{l l e}$ UAU relative to U6 of MDA (a) or

1065 HCC1806 (b) Parental cells with control or overexpression of tRNA ${ }^{\text {lle }}$ UAU.

1066 (c) Northern blot quantification of tRNA ${ }^{\text {lle }}$ UAU relative to U6 of LM2 cells depleted of tRNA ${ }^{\text {lle }}$ UAU via CRISPR with Guide 1 or 2 versus control.

1068 (d) tRNA ${ }^{1 \mathrm{le}}{ }_{\text {GAU }}$ quantification by specific tRNA ${ }^{\text {lle }}$ GAU probe RT-qPCR normalized to $18 S$ probes of LM2 cells with control or overexpression of tRNA ${ }^{\mathrm{lle}}{ }_{G A U}$. $(e, f)$ tRNA ${ }^{l l e}{ }_{G A U}$ quantification by specific tRNA ${ }^{l l e}{ }_{G A U}$ probe RT-qPCR normalized to 18S probes MDA Parental CRISPRi cells with guides targeting either control or tRNA ${ }^{11}{ }_{\text {GAU }}(e)$ or or shRNA targeting control or tRNA ${ }^{1 l e}{ }_{\text {GAU }}(f)$. Two sided un-paired student t-tests performed, $p$-values representated as ${ }^{*},{ }^{* *}$, ${ }^{* * *}$ as $p<0.05, p<0.01$, $p<0.001$. 

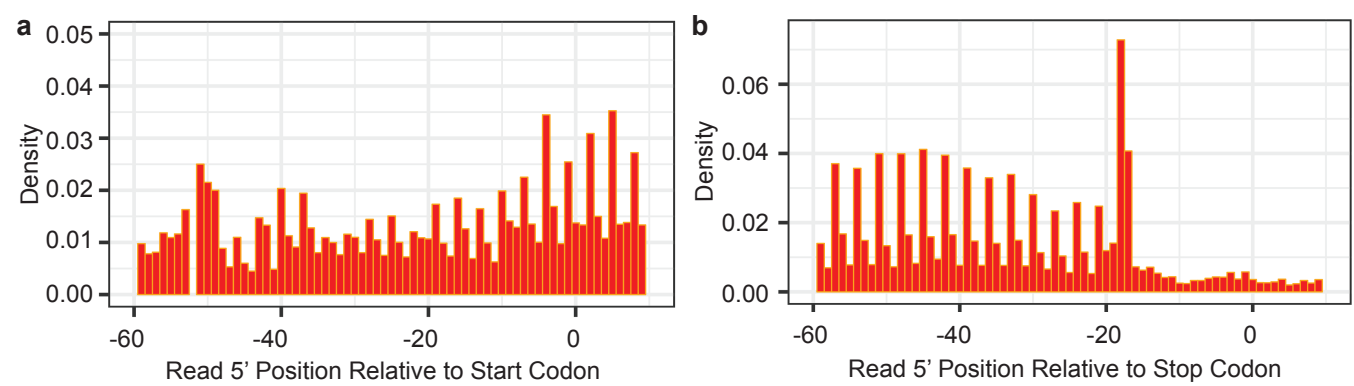

c

d
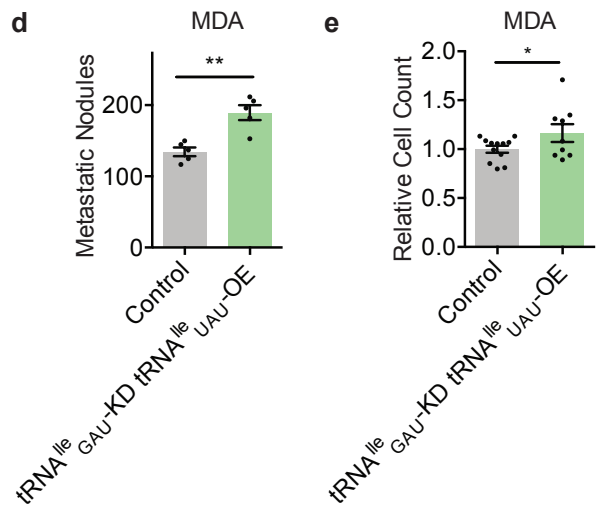

f
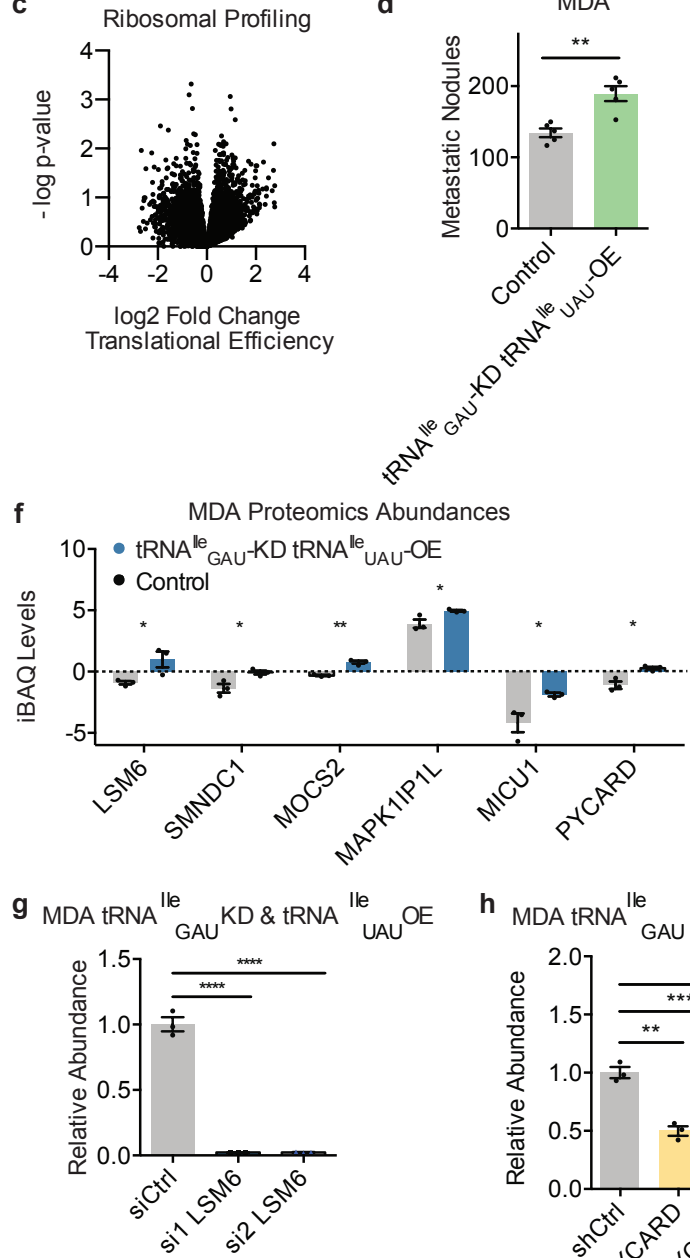

h MDA tRNA ${ }_{\text {GAU }}^{\text {lle }}$ KD \& tRNA ${ }_{\text {UaU }}^{\text {lle }}$ OE

Supplementary Figure 3 - Downstream effectors of tRNA ${ }^{\text {lle }}{ }_{\text {GAU }}$ depletion and

1076

1077 tRNA ${ }^{l l e}$ UAU Overexpression mediate increased growth under metastatic stress conditions

$(a, b)$. Histogram of read coverage to demonstrate 3 nucleotide periodicity of the coding sequence with respect to the start (a) and stop codon (b) of the reading

1080 frame.

1081

1082

1083

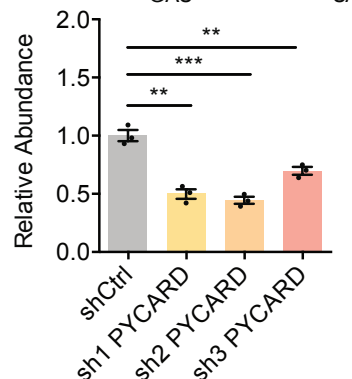

(c) Volcano plot representing log2 fold change vs. $-\log p$ value of translational efficiency from ribosomal profiling of MDA tRNA ${ }^{1 l e}{ }_{G A U}$ depleted and TRNA ${ }^{1 / \mathrm{e}}$ UAU overexpression cells versus control. 
1084 (d) Quantification of lung metastatic nodules post extraction after tail vein injection 1085 of $1 \times 10^{5}$ MDA tRNA ${ }^{l l e}$ GAU depleted and tRNA ${ }^{\text {lle }}$ UAU overexpression cells versus 1086 control.

1087 (e) Relative cell counts of MDA MB 231 control \& tRNA ${ }^{l l e}$ GAU depletion tRNA ${ }^{\text {lle }}$ UAU 1088 overexpression cells after 5 days. Two-sided un-paired student's t-tests performed, p-values represented as *, ${ }^{* *}$ as $p<0.05, p<0.01$, respectively. (f) iBAQ values of six candidate downstream effectors, measured by label free quantification mass spectrometry; 3 biological replicates each.

(g) RT-qPCR quantification of LSM6 cDNA levels normalized to GAPDH on Day 2 of siRNA transfection. (h) RT-qPCR quantification of PYCARD cDNA levels normalized to GAPDH. Twosided un-paired student's t-tests performed, $\mathrm{p}$-values represented ${ }^{* *},{ }^{* * *},{ }^{* * *}$ as $p<0.01, p<0.001, p, 0.0001$, respectively.
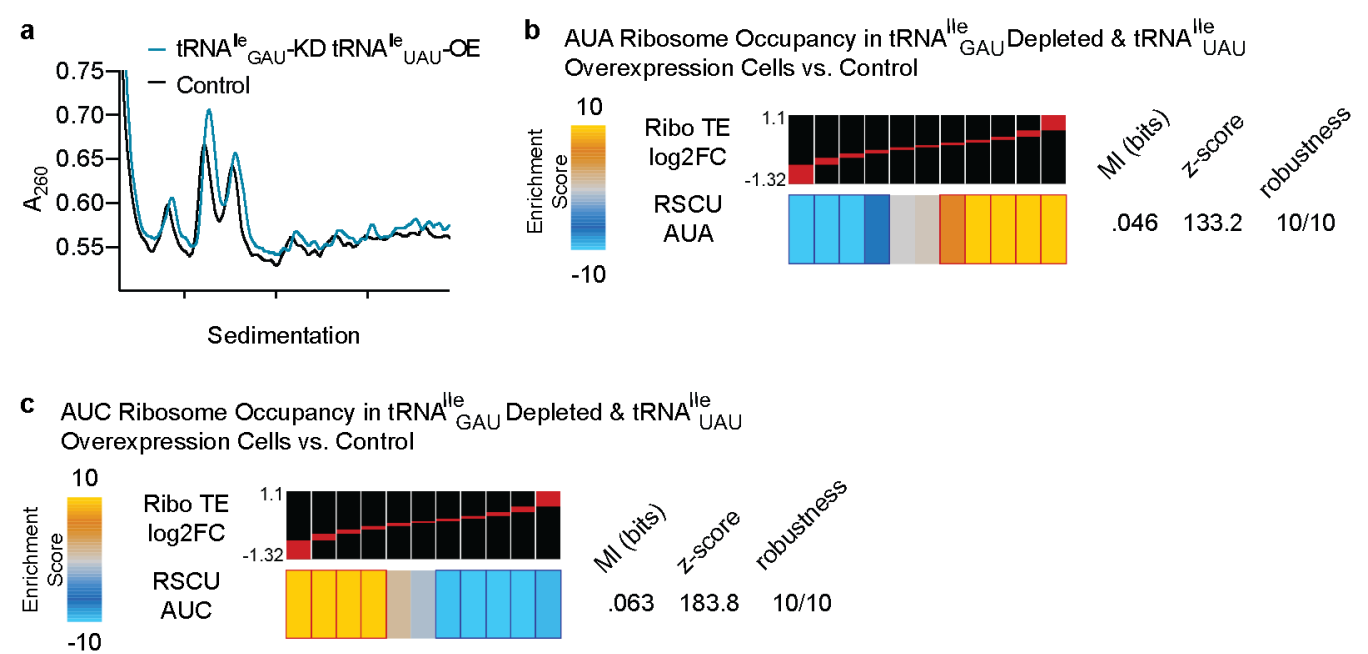

1098 Supplementary Figure 4 - Ribosomal profiling of MDA cells concurrently modulated with tRNA ${ }^{\mathrm{Ile}}{ }_{\text {GAU }}$ depletion \& tRNA ${ }^{\text {lle }}$ UAU overexpression. (a) Polysome traces of two representative samples, measured by UV spectrometry.

(b) Genes with a high abundance of AUA codons were significantly enriched among genes significantly upregulated in ribosomal protected fragments (corrected for their transcript changes) in tRNA ${ }^{l l e}{ }_{G A U}$ depleted tRNA ${ }^{\text {lle }}$ UAU overexpression cells versus control MDA MB 231 cells, measured by ribosomal profiling. The statistical significance of these enrichments was assessed using mutual-information calculations and associated $\mathrm{Z}$ score (based on randomized input vectors). Also included is the $x^{2} p$ value for the associated contingency table. The heatmap was generated using the -log of the hypergeometric $p$ value for enrichment and log of $p$ value for depletion (collectively termed the enrichment score). The red and dark-blue borders indicate the statistical significance of the calculated hypergeometric $p$ values.

1114 for by relative synonymous codon usage score (RSCU). 